\title{
THE DECOMPOSITION METHOD FOR LINEAR, ONE-DIMENSIONAL, TIME-DEPENDENT PARTIAL DIFFERENTIAL EQUATIONS
}

D. LESNIC

Received 2 December 2005; Revised 15 May 2006; Accepted 20 June 2006

The analytical solutions for linear, one-dimensional, time-dependent partial differential equations subject to initial or lateral boundary conditions are reviewed and obtained in the form of convergent Adomian decomposition power series with easily computable components. The efficiency and power of the technique are shown for wide classes of equations of mathematical physics.

Copyright (c) 2006 Hindawi Publishing Corporation. All rights reserved.

\section{Introduction}

We consider linear, one-dimensional, time-dependent partial differential equations (PDEs) of the form

$$
\sum_{n=0}^{N} \alpha_{n}(x, t) \frac{\partial^{n} u}{\partial t^{n}}=\sum_{m=1}^{M} \beta_{m}(x, t) \frac{\partial^{m} u}{\partial x^{m}}(x, t)+f(x, t), \quad(x, t) \in \Omega \subset \mathbb{R}^{2},
$$

where $\left(\alpha_{n}\right)_{n=\overline{0, N}},\left(\beta_{m}\right)_{m=\overline{1, M}}$ are given coefficients, $\alpha_{n} \neq 0, \beta_{M} \neq 0$, and $N, M$ are positive integers. Associated with (1.1), we can consider the initial conditions

$$
\frac{\partial^{n} u}{\partial t^{n}}(x, 0)=g_{n}(x), \quad n=\overline{0,(N-1)}, x \in \mathbb{R},
$$

or the lateral (Cauchy) boundary conditions

$$
\frac{\partial^{m} u}{\partial x^{m}}(0, t)=f_{m}(t), \quad m=\overline{0,(M-1)}, t \in \mathbb{R}
$$

When the initial conditions (1.2) are imposed, $\Omega=\mathbb{R} \times(0, \infty)$; whilst when the lateral boundary conditions (1.3) are imposed, $\Omega=(0, \infty) \times \mathbb{R}$. Further, we assume that the functions $f,\left(\alpha_{n}\right)_{n=\overline{0, N}},\left(\beta_{m}\right)_{m=\overline{1, M}},\left(g_{n}\right)_{n=\overline{0,(N-1)}}$, and $\left(f_{m}\right)_{m=\overline{1,(M-1)}}$ are such that problems (1.1) and (1.2) and (1.1) and (1.3) have a solution. 
In recent years, the Adomian decomposition method (ADM) has been applied to wide classes of stochastic and deterministic problems in many interesting mathematical and physical areas, [5, 6]. For linear PDEs, this method is similar to the method of successive approximations (Picard's iterations), whilst for nonlinear PDEs, is similar to the homotopy or imbedding method, [24]. The ADM provides analytical, verifiable, and rapidly convergent approximations which yield insight into the character and behaviour of the solution just as in the closed-form solution. In this study, we review and develop new applications of the ADM for solving linear PDEs of the type (1.1) subject to the initial conditions (1.2), or to the lateral boundary conditions (1.3).

A wide range of linear PDEs, which have very important practical applications in mathematical physics, (see [35]), are investigated which include the advection equation (Section 4.1), the heat equation (Section 4.2), the wave equation (Section 4.3), the KdV equation (Section 4.4), and the Euler-Bernoulli equation (Section 4.5). Extensions to systems of linear PDEs and nonlinear PDEs, (see [20]) are presented in Sections 5 and 6, respectively. Finally, conclusions are presented in Section 7.

\section{Adomian's decomposition method}

First, let us define the following differential operators:

$$
\begin{aligned}
& G_{n}=\frac{\partial^{n}}{\partial t^{n}}, \quad n=\overline{0, N}, \\
& F_{m}=\frac{\partial^{m}}{\partial x^{m}}, \quad m=\overline{0, M},
\end{aligned}
$$

with the convention that $G_{0}=F_{0}=I=$ the identity operator.

Then (1.1)-(1.3) can be rewritten as

$$
\begin{aligned}
\sum_{n=0}^{N} \alpha_{n}(x, t) G_{n} u(x, t) & =\sum_{m=1}^{M} \beta_{m}(x, t) F_{m} u(x, t)+f(x, t), \quad(x, t) \in \Omega, \\
G_{n}(x, 0) & =g_{n}(x), \quad n=\overline{0,(N-1)}, x \in \mathbb{R}, \\
F_{m}(0, t) & =f_{m}(t), \quad m=\overline{0,(M-1)}, t \in \mathbb{R} .
\end{aligned}
$$

Now let us formally define the left-inverse integral operators

$$
\begin{aligned}
& G_{N}^{-1}=\int_{0}^{t_{0}=t} \int_{0}^{t_{1}} \cdots \int_{0}^{t_{N-1}} d t_{N} \cdots d t_{1}, \\
& F_{M}^{-1}=\int_{0}^{x_{0}=x} \int_{0}^{x_{1}} \cdots \int_{0}^{x_{M-1}} d x_{M} \cdots d x_{1} .
\end{aligned}
$$


Applying (2.5) to (2.2) and using (2.3), and (2.6) to (2.2) and using (2.4), we obtain

$$
\begin{aligned}
u(x, t)= & G_{N}^{-1}\left(\frac{f(x, t)}{\alpha_{N}(x, t)}\right)+\sum_{n=0}^{N-1} \frac{t^{n}}{n !} g_{n}(x)+\sum_{m=1}^{M} G_{N}^{-1}\left(\frac{\beta_{m}(x, t)}{\alpha_{N}(x, t)} F_{m} u(x, t)\right) \\
& -\sum_{n=0}^{N-1} G_{N}^{-1}\left(\frac{\alpha_{n}(x, t)}{\alpha_{N}(x, t)} G_{n} u(x, t)\right), \\
u(x, t)= & -F_{M}^{-1}\left(\frac{f(x, t)}{\beta_{M}(x, t)}\right)+\sum_{m=0}^{M-1} \frac{x^{m}}{m !} f_{m}(t)+\sum_{n=0}^{N} F_{M}^{-1}\left(\frac{\alpha_{n}(x, t)}{\beta_{M}(x, t)} G_{n} u(x, t)\right) \\
& -\sum_{m=1}^{M-1} F_{M}^{-1}\left(\frac{\beta_{m}(x, t)}{\beta_{M}(x, t)} F_{m} u(x, t)\right),
\end{aligned}
$$

respectively, where the last term in (2.8) vanishes if $M=1$.

Using the ADM (see [6]), we define the following relationships for (2.7) and (2.8), namely,

$$
\begin{gathered}
u_{0}(x, t)=G_{N}^{-1}\left(\frac{f(x, t)}{\alpha_{N}(x, t)}\right)+\sum_{l=0}^{N-1} \frac{t^{l}}{l !} g_{l}(x), \\
u_{k+1}(x, t)=\left[\sum_{m=1}^{M} G_{N}^{-1}\left(\frac{\beta_{m}(x, t)}{\alpha_{N}(x, t)} F_{m}\right)-\sum_{n=0}^{N-1} G_{N}^{-1}\left(\frac{\alpha_{n}(x, t)}{\alpha_{N}(x, t)} G_{n}\right)\right] u_{k}(x, t), \quad k \geq 0, \\
u_{0}(x, t)=-F_{M}^{-1}\left(\frac{f(x, t)}{\beta_{M}(x, t)}\right)+\sum_{l=0}^{M-1} \frac{x^{l}}{l !} f_{l}(t), \\
u_{k+1}(x, t)=\left[\sum_{n=0}^{N} F_{M}^{-1}\left(\frac{\alpha_{n}(x, t)}{\beta_{M}(x, t)} G_{n}\right)-\sum_{m=1}^{M-1} F_{M}^{-1}\left(\frac{\beta_{m}(x, t)}{\beta_{M}(x, t)} F_{m}\right)\right] u_{k}(x, t), \quad k \geq 0,
\end{gathered}
$$

respectively. Then we expect that

$$
u(x, t)=\sum_{k=0}^{\infty} u_{k}(x, t)
$$

or if we define the sequence of partial sums

$$
\phi_{K}(x, t)=\sum_{k=0}^{K} u_{k}(x, t), \quad K \geq 0,
$$

then $\lim _{K \rightarrow \infty} \phi_{K}(x, t)=u(x, t)$.

Equation (2.9), via (2.11), gives the solution of problem (1.1) and (1.2) in $\Omega=\mathbb{R} \times$ $(0, \infty)$, whilst (2.10), via (2.11), gives the solution of problem (1.1) and (1.3) in $\Omega=$ $(0, \infty) \times \mathbb{R}$. 
4 The decomposition method for linear PDEs

\section{A special case}

We consider the special case of (1.1) with $\alpha_{n}=0$ for $n=\overline{0,(N-1)}, \beta_{m}=0$ for $m=$ $\overline{0,(M-1)}, f=0, \alpha_{N}, \beta_{M}$ nonzero constants, given by

$$
\alpha_{N} \frac{\partial^{N} u}{\partial t^{N}}(x, t)=\beta_{M} \frac{\partial^{M} u}{\partial x^{M}}(x, t), \quad(x, t) \in \Omega .
$$

Then (2.9) and (2.10) simplify to

$$
\begin{array}{ll}
u_{0}(x, t)=\sum_{l=0}^{N-1} \frac{t^{l}}{l !} g_{l}(x), & u_{k+1}(x, t)=\frac{\beta_{M}}{\alpha_{N}} G_{N}^{-1} F_{M} u_{k}(x, t), \quad k \geq 0, \\
u_{0}(x, t)=\sum_{l=0}^{M-1} \frac{t^{l}}{l !} f_{l}(t), & u_{k+1}(x, t)=\frac{\alpha_{N}}{\beta_{M}} F_{M}^{-1} G_{N} u_{k}(x, t), \quad k \geq 0,
\end{array}
$$

respectively.

Solving (3.2), we obtain

$$
\begin{aligned}
& u_{k}(x, t)=\left(\frac{\beta_{M}}{\alpha_{N}}\right) \sum_{l=0}^{k} \frac{t^{l+N k}}{(l+N k) !} g_{l}^{(M k)}(x), \quad k \geq 0, \\
& u_{k}(x, t)=\left(\frac{\alpha_{N}}{\beta_{M}}\right) \sum_{l=0}^{k} \frac{x^{l+M k}}{(l+M k) !} f_{l}^{(N k)}(t), \quad k \geq 0,
\end{aligned}
$$

respectively.

Then (2.11) gives explicitly the ADM partial $t$-solution of (1.2) and (3.1) as

$$
u(x, t)=\sum_{k=0}^{\infty}\left(\frac{\beta_{M}}{\alpha_{N}}\right)^{k} \sum_{l=0}^{N-1} \frac{t^{l+N k}}{(l+N k) !} g_{l}^{(M k)}(x), \quad(x, t) \in \mathbb{R} \times[0, \infty),
$$

and the ADM partial $x$-solution of (1.3) and (3.1) as

$$
u(x, t)=\sum_{k=0}^{\infty}\left(\frac{\alpha_{N}}{\beta_{M}}\right)^{k} \sum_{l=0}^{M-1} \frac{x^{l+M k}}{(l+M k) !} f_{l}^{(N k)}(t), \quad(x, t) \in[0, \infty) \times \mathbb{R} .
$$

These solutions will be equal only when the compatibility conditions

$$
f_{m}(t)=\sum_{k=0}^{\infty}\left(\frac{\beta_{M}}{\alpha_{N}}\right)^{k} \sum_{l=0}^{N-1} \frac{t^{l+N k}}{(l+N k) !} g_{l}^{(M k+m)}(0), \quad m=\overline{0,(M-1)}, t \in[0, \infty),
$$

and the partial $x$-solution of (1.3) and (3.1) as

$$
g_{n}(x)=\sum_{k=0}^{\infty}\left(\frac{\alpha_{N}}{\beta_{M}}\right)^{k} \sum_{l=0}^{M-1} \frac{x^{l+M k}}{(l+M k) !} f_{l}^{(N k+n)}(0), \quad n=\overline{0,(N-1)}, x \in[0, \infty),
$$

hold. 


\section{Applications}

Without loss of generality, we may assume that $N \geq M$.

4.1. The advection equation $(N=M=1)$. In this application, we consider the timedependent spread of contaminants in moving fluids, which, in the simplest case, is governed by the one-dimensional linear advection equation

$$
\frac{\partial u}{\partial t}(x, t)=\beta_{1} \frac{\partial u}{\partial x}(x, t), \quad(x, t) \in \Omega
$$

where $\beta_{1}$ is the constant coefficient of advection, which corresponds to the case $N=M=$ $1, \alpha_{1}=1$ in (3.1).

If (4.1) is solved subject to the initial condition

$$
u(x, 0)=g_{0}(x), \quad x \in \mathbb{R},
$$

then (3.4) gives the ADM partial $t$-solution

$$
u(x, t)=\sum_{k=0}^{\infty} \frac{\left(\beta_{1} t\right)^{k}}{k !} g_{0}^{(k)}(x), \quad(x, t) \in \mathbb{R} \times[0, \infty),
$$

whilst if (4.1) is solved subject to the boundary condition

$$
u(0, t)=f_{0}(t), \quad t \in \mathbb{R},
$$

then (3.5) gives the ADM partial $x$-solution (see [8])

$$
u(x, t)=\sum_{k=0}^{\infty} \frac{x^{k}}{\beta_{1}^{k} k !} f_{0}^{(k)}(t), \quad(x, t) \in[0, \infty) \times \mathbb{R} .
$$

Example 4.1. Taking $\beta_{1}=1, g_{0}(x)=x, f_{0}(t)=t$, then both the ADM partial solutions (4.3) and (4.5) give, with only two terms $u=u_{0}+u_{1}$ in the decomposition series (2.11), the exact solution $u(x, t)=x+t$ of problem (4.1), (4.2), and (4.4). It is worth noting that this solution can also be obtained by using the ADM complete solution (see [1]) based on the recursive relationship

$$
\begin{gathered}
u_{0}(x, t)=\frac{1}{2}\left(f_{0}(t)+g_{0}(x)\right)=\frac{x+t}{2}, \\
u_{k+1}(x, t)=\frac{1}{2}\left[G_{1}^{-1} F_{1}+F_{1}^{-1} G_{1}\right] u_{k}(x, t)=\frac{x+t}{2^{k+1}}, \quad k \geq 0,
\end{gathered}
$$

using (2.11), that is,

$$
u(x, t)=\sum_{k=0}^{\infty} u_{k}(x, t)=\sum_{k=0}^{\infty} \frac{x+t}{2^{k+1}}=x+t
$$


6 The decomposition method for linear PDEs

4.1.1. The reaction-advection equation. We consider the linear reaction-advection equation

$$
\alpha_{0} u(x, t)+\frac{\partial u}{\partial t}(x, t)=\beta_{1} \frac{\partial u}{\partial x}(x, t), \quad(x, t) \in \Omega,
$$

where $\beta_{1}, \alpha_{0}$ are constants, which corresponds to the case $N=M=1, \alpha_{1}=1, f=0$ in $(1.1)$.

If (4.8) is solved subject to the initial condition (4.2), then (2.9) gives

$$
u_{0}(x, t)=g_{0}(x), \quad u_{k+1}(x, t)=\left(\beta_{1} G_{1}^{-1} F_{1}-\alpha_{0} G_{1}^{-1}\right) u_{k}(x, t), \quad k \geq 0 .
$$

Calculating a few terms in (4.9), we obtain

$$
u_{1}(x, t)=\left(\beta_{1} g_{0}^{\prime}(x)-\alpha_{0} g_{0}(x)\right) t, \quad u_{2}(x, t)=\left(\beta_{1}^{2} g_{0}^{\prime \prime}(x)-2 \beta_{1} \alpha_{0} g_{0}^{\prime}(x)+\alpha_{0}^{2} g_{0}(x)\right) \frac{t^{2}}{2 !},
$$

and in general

$$
u_{k}(x, t)=\frac{t^{k}}{k !} \sum_{l=0}^{k} C_{k}^{l} \beta_{1}^{k-l}\left(-\alpha_{0}\right)^{l} g_{0}^{(l)}(x), \quad k \geq 0,
$$

where $C_{k}^{l}=k ! / l !(k-l) !$. Then (2.11) gives the ADM partial $t$-solution of problem (4.2) and (4.8) as

$$
u(x, t)=\sum_{k=0}^{\infty} \frac{t^{k}}{k !} \sum_{l=0}^{k} C_{k}^{l} \beta_{1}^{k-l}\left(-\alpha_{0}\right)^{l} g_{0}^{(l)}(x), \quad(x, t) \in \mathbb{R} \times[0, \infty) .
$$

If now (4.8) is solved subject to the boundary condition (4.4), similarly as above one obtains the ADM partial $x$-solution given by

$$
u(x, t)=\sum_{k=0}^{\infty} \frac{x^{k}}{\beta_{1}^{k} k !} \sum_{l=0}^{k} C_{k}^{l} \alpha_{0}^{l} f_{0}^{(l)}(t), \quad(x, t) \in[0, \infty) \times \mathbb{R} .
$$

4.2. The heat (diffusion) equation $(N=1, M=2)$. Consider the linear heat equation

$$
\frac{\partial u}{\partial t}(x, t)=\beta_{2} \frac{\partial^{2}}{\partial x^{2}}(x, t), \quad(x, t) \in \Omega,
$$

where $\beta_{2}>0$ is the constant coefficient of diffusion, which corresponds to the case $N=1$, $M=2, \alpha_{1}=1$ in (3.1).

If (4.14) is solved subject to the initial condition (4.2), then (3.4) gives the ADM partial $t$-solution of the characteristic Cauchy problem for the heat equation, namely,

$$
u(x, t)=\sum_{k}^{\infty} \frac{\left(\beta_{2} t\right)^{k}}{k !} g_{0}^{(2 k)}(x), \quad(x, t) \in \mathbb{R} \times[0, \infty),
$$


whilst if (4.14) is solved subject to the lateral boundary conditions

$$
u(0, t)=f_{0}(t), \quad \frac{\partial u}{\partial x}(0, t)=f_{1}(t), \quad t \in \mathbb{R},
$$

then (3.5) gives the ADM partial $x$-solution of the noncharacteristic Cauchy problem for the heat equation (see [33])

$$
u(x, t)=\sum_{k=0}^{\infty} \frac{1}{\beta_{2}^{k}}\left[\frac{f_{0}^{(k)}(t)}{(2 k) !} x^{2 k}+\frac{f_{1}^{(k)}(t)}{(2 k+1) !} x^{2 k+1}\right], \quad(x, t) \in[0, \infty) \times \mathbb{R} .
$$

The solution (4.15) represents a simplified improvement over the Green formula and was previously obtained in [15] using the method of separating variables.

Particular examples of the Cauchy problems (4.14), and (4.2) or (4.16), solved using the ADM, can be found in $[2,3,13,31,39,45,47]$.

4.2.1. The reaction-diffusion equation. We consider the biological interpretation of (4.14) with a linear source

$$
\alpha_{0} u(x, t)+\frac{\partial u}{\partial t}(x, t)=\beta_{2} \frac{\partial^{2}}{\partial x^{2}}(x, t), \quad(x, t) \in \Omega
$$

where $\beta_{2}>0, \alpha_{0}$ are constants, which corresponds to the case $N=1, M=2, \beta_{1}=f=0$, $\alpha_{1}=1$ in (1.1). In contrast to the simple diffusion $\left(\alpha_{0}=0\right.$, see (4.14)), when reaction kinetics and diffusion are coupled through the term $\alpha_{0} u$, travelling waves of chemical concentration $u$ may exist and can affect a biological change much faster than the straight diffusional process, see [34].

If (4.18) is solved subject to the initial condition (4.2) then, similarly as in Section 4.1.1, one obtains the ADM partial $t$-solution given by

$$
u(x, t)=\sum_{k=0}^{\infty} \frac{t^{k}}{k !} \sum_{l=0}^{k} C_{k}^{l} \beta_{2}^{k-l}\left(-\alpha_{0}\right)^{l} g_{0}^{(2 l)}(x), \quad(x, t) \in \mathbb{R} \times[0, \infty) .
$$

On the other hand if (4.18) is solved subject to the boundary conditions (4.16), then (2.10) gives

$$
u_{0}(x, t)=f_{0}(t)+x f_{1}(t), \quad u_{k+1}(x, t)=\frac{1}{\beta_{2}}\left(\alpha_{0} F_{2}^{-1}+F_{2}^{-1} G_{1}\right) u_{k}(x, t), \quad k \geq 0 .
$$

Calculating a few terms in (4.20), we obtain

$$
\begin{gathered}
u_{1}(x, t)=\frac{1}{\beta_{2}}\left[\left(f_{0}^{\prime}(t)+\alpha_{0} f_{0}(t)\right) \frac{x^{2}}{2 !}+\left(f_{1}^{\prime}(t)+\alpha_{0} f_{1}(t)\right) \frac{x^{3}}{3 !}\right], \\
u_{2}(x, t)=\frac{1}{\beta_{2}^{2}}\left[\left(f_{0}^{\prime \prime}(t)+2 \alpha_{0} f_{0}(t) f_{0}^{\prime}(t)+\alpha_{0}^{2} f_{0}(t)\right) \frac{x^{4}}{4 !}+\left(f_{1}^{\prime \prime}(t)+2 \alpha_{0} f_{1}(t) f_{1}^{\prime}(t)+\alpha_{0}^{2} f_{1}(t)\right) \frac{x^{5}}{5 !}\right],
\end{gathered}
$$


8 The decomposition method for linear PDEs

and in general

$$
u_{k}(x, t)=\frac{1}{\beta_{2}^{k}}\left[\frac{x^{2 k}}{(2 k) !} \sum_{l=0}^{k} C_{k}^{l} \alpha_{0}^{l}\left(f_{0}^{(l)}(t)+\frac{x}{2 k+1} f_{1}^{(l)}(t)\right)\right], \quad k \geq 0
$$

Then (2.11) gives the ADM partial $x$-solution of problem (4.2) and (4.18) as given by

$$
u(x, t)=\sum_{k=0}^{\infty} \frac{x^{2 k}}{\beta_{2}^{k}(2 k) !} \sum_{l=0}^{k} C_{k}^{l} \alpha_{0}^{l}\left(f_{0}^{(l)}(t)+\frac{x}{2 k+1} f_{1}^{(l)}(t)\right), \quad(x, t) \in[0, \infty) \times \mathbb{R} .
$$

For particular cases of $f_{0}, f_{1}$, and $g_{0}$, one can calculate the series (4.19) and (4.23) explicitly, see [36].

4.2.2. The advection-diffusion equation. Taking $N=1, M=2, \alpha_{0}=f=0, \alpha_{1}=1$ in (1.1), we obtain the advection-diffusion equation

$$
\frac{\partial u}{\partial t}(x, t)=\beta_{2} \frac{\partial^{2} u}{\partial x^{2}}(x, t)+\beta_{1} \frac{\partial u}{\partial x}(x, t), \quad(x, t) \in \Omega,
$$

which arises in advective-diffusive flows when analysing the mechanics governing the release of hormones from secretory cells in response to a stimulus in a medium, flowing past the cells and through a diffusion column, see [38]. In (4.24), $\beta_{2}>0$ is the diffusion coefficient, $u$ is the concentration of hormones, and $-\beta_{1}>0$ is the flow velocity down the column. A similar situation arises in forced convection cooling of flat electronic substrates, (see [19]) or in the dispersion of pollutants in rivers.

For $\beta_{1}=$ constant, the ADM partial $t$-solution of problem (4.2) and (4.24) is given by (see $[32])$

$$
u(x, t)=\exp \left(-\frac{\beta_{1} x}{2 \beta_{2}}-\frac{\beta_{1}^{2} t}{4 \beta_{2}}\right) \sum_{k=0}^{\infty} \frac{\left(\beta_{2} t\right)^{k}}{k !} \theta_{0}^{(2 k)}(x), \quad(x, t) \in \mathbb{R} \times[0, \infty),
$$

where

$$
\theta_{0}(x)=g_{0}(x) \exp \left(\frac{\beta_{1} x}{2 \beta_{2}}\right), \quad x \in \mathbb{R}
$$

whilst the ADM partial $x$-solution of problem (4.16) and (4.24) is given by

$$
u(x, t)=\exp \left(-\frac{\beta_{1} x}{2 \beta_{2}}-\frac{\beta_{1}^{2} t}{4 \beta_{2}}\right) \sum_{k=0}^{\infty} \frac{1}{\beta_{2}^{k}}\left[\frac{x^{2 k}}{(2 k) !} \psi_{0}^{(k)}(t)+\frac{x^{2 k+1}}{(2 k+1) !} \psi_{1}^{(k)}(t)\right], \quad(x, t) \in[0, \infty) \times \mathbb{R}
$$

where

$$
\psi_{0}(t)=f_{0}(t) \exp \left(\frac{\beta_{1}^{2} t}{4 \beta_{2}}\right), \quad \psi_{1}(t)=\left(f_{1}(t)+\frac{\beta_{1}}{2 \beta_{2}} f_{0}(t)\right) \exp \left(\frac{\beta_{1}^{2} t}{4 \beta_{2}}\right), \quad t \in \mathbb{R} .
$$


Example 4.2. Taking $\beta_{1}=-1, \beta_{2}=1$, then (4.24) becomes

$$
\frac{\partial u}{\partial t}(x, t)=\frac{\partial^{2} u}{\partial x^{2}}(x, t)-\frac{\partial u}{\partial x}(x, t), \quad(x, t) \in \Omega
$$

and consider the initial and boundary conditions

$$
\begin{gathered}
u(x, 0)=e^{x}-x=g_{0}(x), \quad x \in \mathbb{R}, \\
u(0, t)=1+t=f_{0}(t), \quad \frac{\partial u}{\partial x}(0, t)=0=f_{1}(t), \quad t \in \mathbb{R} .
\end{gathered}
$$

Then using (4.26) and (4.28), we obtain

$$
\begin{gathered}
\theta_{0}(x)=e^{x / 2}-x e^{-x / 2}, \quad x \in \mathbb{R}, \\
\psi_{0}(t)=(1+t) e^{t / 4}, \quad \psi_{1}(t)=-\frac{(1+t)}{2} e^{t / 4}, \quad t \in \mathbb{R} .
\end{gathered}
$$

Using Leibniz's rule of product differentiation, we obtain

$$
\begin{gathered}
\theta_{0}^{(k)}(x)=2^{-k}\left(e^{x / 2}+(2 k-x) e^{-x / 2}\right), \quad k \geq 0, \\
\psi_{0}^{(k)}(t)=\frac{(1+4 k+t)}{4^{k}} e^{t / 4}, \quad \psi_{1}^{(k)}(t)=-\frac{(1+4 k+t)}{2 \cdot 4^{k}} e^{t / 4}, \quad k \geq 0 .
\end{gathered}
$$

Introducing (4.33) into (4.25), we obtain the ADM partial $t$-solution of problem (4.29) and (4.30) as

$$
\begin{aligned}
u(x, t) & =e^{(x / 2-t / 4)} \sum_{k=0}^{\infty} \frac{4^{-k} t^{k}}{k !}\left(e^{x / 2}+(4 k-x) e^{-x / 2}\right) \\
& =e^{x}-x+e^{-t / 4} \sum_{k=1}^{\infty} \frac{4^{1-k} t^{k}}{(k-1) !}=e^{x}-x+t, \quad(x, t) \in \mathbb{R} \times[0, \infty) .
\end{aligned}
$$

Also introducing (4.34) into (4.27), we obtain the ADM partial $x$-solution of problem (4.29) and (4.31) as

$$
\begin{aligned}
u(x, t) & =e^{x / 2} \sum_{k=0}^{\infty} 4^{-k}\left[(1+4 k+t) \frac{x^{2 k}}{(2 k) !}-\frac{(1+4 k+t)}{2} \frac{x^{2 k+1}}{(2 k+1) !}\right] \\
& =1+t+e^{x / 2} \sum_{k=0}^{\infty} 4 k\left[\frac{(x / 2)^{2 k}}{(2 k) !}-\frac{(x / 2)^{2 k+1}}{(2 k+1) !}\right]=e^{x}-x+t, \quad(x, t) \in[0, \infty) \times \mathbb{R} .
\end{aligned}
$$

Both the ADM partial series solutions (4.35) and (4.36) yield the exact solution $u(x, t)=e^{x}-x+t$ of problem (4.29)-(4.31) which can be verified through substitution. 
Alternatively, for obtaining the ADM partial $x$-solution, one can use directly the recursive relation (2.10) for problem (4.29) and (4.31) to obtain $u_{0}(x, t)=f_{0}(t)+x f_{1}(t)=$ $1+t, u_{1}(x, t)=F_{2}^{-1}\left(G_{1}+F_{1}\right) u_{0}(x, t)=x^{2} / 2 !, u_{2}(x, t)=F_{2}^{-1}\left(G_{1}+F_{1}\right) u_{1}(x, t)=x^{3} / 3 !$ and in general $u_{k}(x, t)=x^{k+1} /(k+1)$ ! for $k \geq 1$. Then the decomposition series (2.11) gives $u(x, t)=\sum_{k=0}^{\infty} u_{k}(x, t)=1+t+\sum_{k=1}^{\infty}\left(x^{k+1} /(k+1) !\right)=t+e^{x}-x$, as required. Also, for obtaining the ADM partial $t$-solution, one can use directly the recursive relation (2.9) for problem (4.29) and (4.30) to obtain $u_{0}(x, t)=g_{0}(x)=e^{x}-x, u_{1}(x, t)=G_{1}^{-1}\left(F_{2}-F_{1}\right) u_{0}(x$, $t)=t, u_{k}(x, t)=0$ for $k \geq 2$. Thus (2.11) gives the exact solution $u=u_{0}+u_{1}=e^{x}-x+t$ in only two terms. From this, it can be seen that directly applying the ADM to (4.29) produces a faster convergent series solution than (4.35) and (4.36).

4.3. The wave equation $(N=M=2)$. Consider the linear wave equation

$$
\frac{\partial^{2} u}{\partial t^{2}}(x, t)=\beta_{2} \frac{\partial^{2} u}{\partial x^{2}}(x, t), \quad(x, t) \in \Omega,
$$

where $\beta_{2}>0$ is the square of the wave speed, which corresponds to the case $N=M=2$, $\alpha_{2}=1$ in (3.1).

If (4.37) is solved subject to the initial conditions

$$
u(x, 0)=g_{0}(x), \quad \frac{\partial u}{\partial t}(x, 0)=g_{1}(x), \quad x \in \mathbb{R},
$$

then (3.4) gives the ADM partial $t$-solution, (see [42])

$$
u(x, t)=\sum_{k=0}^{\infty} \beta_{2}^{k}\left[g_{0}^{(2 k)}(x) \frac{t^{2 k}}{(2 k) !}+g_{1}^{(2 k)}(x) \frac{t^{2 k+1}}{(2 k+1) !}\right], \quad(x, t) \in \mathbb{R} \times[0, \infty)
$$

whilst if (4.37) is solved subject to the boundary conditions (4.16), then (3.5) gives the partial $x$-solution

$$
u(x, t)=\sum_{k=0}^{\infty} \frac{1}{\beta_{2}^{k}}\left[f_{0}^{(2 k)}(t) \frac{x^{2 k}}{(2 k) !}+f_{1}^{(2 k)}(t) \frac{x^{2 k+1}}{(2 k+1) !}\right], \quad(x, t) \in[0, \infty) \times \mathbb{R}
$$

Particular examples of problem (4.37) and (4.38) solved using the ADM can be found in $[14,17,45,48]$. Note that if we take $\beta_{2}=-1$ in (4.37), we obtain the two-dimensional Laplace equation, which has been dealt with using the ADM elsewhere, see [12].

4.3.1. The telegraph equation. Consider the linear wave (telegraph) equation

$$
\alpha_{1} \frac{\partial u}{\partial t}(x, t)+\frac{\partial^{2} u}{\partial t^{2}}(x, t)=\beta_{2} \frac{\partial^{2} u}{\partial x^{2}}(x, t)+f(x, t), \quad(x, t) \in \Omega,
$$

which corresponds to the case $N=M=2, \alpha_{0}=\beta_{1}=0, \alpha_{2}=1$ in (1.1). 
If (4.41) is solved subject to the initial conditions (4.38), then (2.9) gives

$u_{0}(x, t)=g_{0}(x)+\operatorname{tg}_{1}(x)+G_{2}^{-1} f(x, t), \quad u_{k+1}(x, t)=G_{2}^{-1}\left[\beta_{2} F_{2}-\alpha_{1} G_{1}\right] u_{k}(x, t), \quad k \geq 0$,

whilst if (4.41) is solved subject to the boundary conditions (4.16), then (2.10) gives

$u_{0}(x, t)=f_{t}+x f_{1}(t)-F_{2}^{-1}\left(\frac{f(x, t)}{\beta_{2}}\right), \quad u_{k+1}(x, t)=F_{2}^{-1}\left(\frac{1}{\beta_{2}} G_{2}+\frac{\alpha_{1}}{\beta_{2}} G_{1}\right) u_{k}(x, t), \quad k \geq 0$.

Example 4.3. Take $\beta_{2}=1, \alpha_{1}=3, f(x, t)=3\left(x^{2}+t^{2}+1\right)$ in (4.41) to yield

$$
3 \frac{\partial u}{\partial t}(x, t)+\frac{\partial^{2} u}{\partial t^{2}}(x, t)=\frac{\partial^{2} u}{\partial x^{2}}(x, t)+3\left(x^{2}+t^{2}+1\right), \quad(x, t) \in \Omega,
$$

and consider the initial and boundary conditions

$$
\begin{gathered}
u(x, 0)=x=g_{0}(x), \quad \frac{\partial u}{\partial t}(x, 0)=1+x^{2}=g_{1}(x), \quad x \in \mathbb{R}, \\
u(0, t)=t+\frac{t^{3}}{3}=f_{0}(t), \quad \frac{\partial u}{\partial x}(0, t)=t=f_{1}(t), \quad t \in \mathbb{R} .
\end{gathered}
$$

Calculating the initial term (4.42), we obtain

$$
u_{0}(x, t)=x+t\left(1+x^{2}\right)+\frac{3 t^{2}}{2}\left(x^{2}+1\right)+\frac{t^{4}}{4} .
$$

Observing that the starting term (4.47) can be decomposed into two parts, namely,

$$
u_{0}(x, t)=z_{1}(x, t)+z_{2}(x, t), \quad z_{1}(x, t)=x+t\left(1+x^{2}\right), \quad z_{2}(x, t)=\frac{3 t^{2}}{2}\left(x^{2}+1\right)+\frac{t^{4}}{4},
$$

then a slightly modified recursive algorithm can be used instead of (4.42) (see [43]), namely,

$$
\begin{aligned}
& u_{0}(x, t)=z_{1}(x, t)=x+t\left(1+x^{2}\right), \\
& u_{1}(x, t)=z_{2}(x, t)+G_{2}^{-1}\left[F_{2}-3 G_{1}\right] u_{0}(x, t)=\frac{t^{3}}{3}+\frac{t^{4}}{4}, \\
& u_{2}(x, t)=G_{2}^{-1}\left[F_{2}-3 G_{1}\right] u_{1}(x, t)=\frac{-t^{4}}{4}-\frac{3 t^{5}}{20}, \\
& u_{3}(x, t)=G_{2}^{-1}\left[F_{2}-3 G_{1}\right] u_{2}(x, t)=\frac{3 t^{5}}{20}+\frac{3 t^{6}}{40},
\end{aligned}
$$

and so forth. Then (2.11) gives the ADM partial $t$-solution

$$
u(x, t)=u_{0}+u_{1}+u_{2}+u_{3}+\cdots=x+t\left(1+x^{2}\right)+\frac{t^{3}}{3}, \quad(x, t) \in \mathbb{R} \times[0, \infty),
$$


which can be verified through substitution to be the exact solution of (4.44) and (4.45). The solution (4.50) was also previously obtained in [29] using the classical ADM based on (4.42) with the starting term (4.47), but the calculus in [29] is more complicated.

Calculating now the initial term in (4.43), we obtain

$$
u_{0}(x, t)=t+\frac{t^{3}}{3}+x-\frac{x^{4}}{4}-\frac{3 x^{2}\left(t^{2}+1\right)}{2} .
$$

Similarly as before, by observing that the starting term (4.51) can be decomposed into two parts, namely,

$$
u_{0}(x, t)=z_{1}(x, t)+z_{2}(x, t), \quad z_{1}(x, t)=t+\frac{t^{3}}{3}+x, \quad z_{2}(x, t)=\frac{-x^{4}}{4}-\frac{3 x^{2}\left(t^{2}+1\right)}{2}
$$

we use

$$
\begin{gathered}
u_{0}(x, t)=z_{1}(x, t)=t+\frac{t^{3}}{3}+x, \quad u_{1}(x, t)=z_{2}(x, t)+F_{2}^{-1}\left(G_{2}+3 G_{1}\right) u_{0}(x, t)=x^{2} t-\frac{x^{4}}{4}, \\
u_{2}(x, t)=F_{2}^{-1}\left(G_{2}+3 G_{1}\right) u_{1}(x, t)=\frac{x^{4}}{4}, \quad u_{3}(x, t)=F_{2}^{-1}\left(G_{2}+3 G_{1}\right) u_{2}(x, t)=0
\end{gathered}
$$

and thus $u_{k+1}=F_{2}^{-1}\left(G_{2}+3 G_{1}\right) u_{k}(x, t)=0$ for all $k \geq 2$. Then the exact solution (4.50) of (4.44) and (4.46) is obtained with only three terms $u=u_{0}+u_{1}+u_{2}$ in the decomposition series (2.11). Note that if we take $\beta_{2}=-1$ in (4.41), we obtain the two-dimensional steady-state diffusion equation with advection in the $t$-direction.

4.3.2. The linear Klein-Gordon equation. Consider the linear Klein-Gordon equation

$$
\alpha_{0} u(x, t)+\frac{\partial^{2} u}{\partial t^{2}}(x, t)=\beta_{2} \frac{\partial^{2} u}{\partial x^{2}}(x, t)+f(x, t), \quad(x, t) \in \Omega,
$$

which corresponds to the case $N=M=2, \alpha_{1}=\beta_{1}=0, \alpha_{2}=1$ in (1.1) especially when the linear term $\alpha_{0} u$ in (4.54) is replaced by a nonlinear function, the Klein-Gordon equation plays an important role in the study of solutions in condensed matter physics, (see [16]) and in quantum mechanics and relativistic physics; see [46].

If (4.54) is solved subject to the initial conditions (4.38), then (2.9) gives

$$
u_{0}(x, t)=g_{0}(x)+\operatorname{tg}_{1}(x)+G_{2}^{-1} f(x, t), u_{k+1}(x, t)=G_{2}^{-1}\left[\beta_{2} F_{2}-\alpha_{0} I\right] u_{k}(x, t), \quad k \geq 0,
$$

whilst if (4.54) is solved subject to the boundary conditions (4.16), then (2.10) gives

$$
\begin{gathered}
u_{0}(x, t)=f_{0}(t)+x f_{1}(t)-F_{2}^{-1}\left(\frac{f(x, t)}{\beta_{2}}\right), \\
u_{k+1}(x, t)=F_{2}^{-1}\left(\frac{1}{\beta_{2}} G_{2}+\frac{\alpha_{0}}{\beta_{2}} I\right) u_{k}(x, t), \quad k \geq 0 .
\end{gathered}
$$


Example 4.4. Take $\beta_{2}=1, \alpha_{0}=-1, f=0$ in (4.54) to yield

$$
\frac{\partial^{2} u}{\partial t^{2}}(x, t)-u(x, t)=\frac{\partial^{2} u}{\partial x^{2}}(x, t), \quad(x, t) \in \Omega,
$$

and consider the initial and boundary conditions

$$
\begin{gathered}
u(x, 0)=1+\sin (x)=g_{0}(x), \quad \frac{\partial u}{\partial t}(x, 0)=0=g_{1}(x), \quad x \in \mathbb{R}, \\
u(0, t)=\cosh (t)=f_{0}(t), \quad \frac{\partial u}{\partial x}(0, t)=1=f_{1}(t), \quad t \in \mathbb{R} .
\end{gathered}
$$

Applying (4.55), we obtain

$$
u_{0}(x, t)=1+\sin (x), \quad u_{1}(x, t)=G_{2}^{-1}\left[F_{2}+I\right] u_{0}(x, t)=\frac{t^{2}}{2 !},
$$

and in general (see [22])

$$
u_{k+1}(x, t)=G_{2}^{-1}\left[F_{2}+I\right] u_{k}(x, t)=\frac{t^{2 k+2}}{(2 k+2) !}, \quad \forall k \geq 0 .
$$

Then (2.11) gives the ADM partial $t$-solution

$$
u(x, t)=\sin (x)+\sum_{k=0}^{\infty} \frac{t^{2 k}}{(2 k) !}=\sin (x)+\cosh (t), \quad(x, t) \in \mathbb{R} \times[0, \infty),
$$

which can be verified through substitution to be the exact solution of (4.57) and (4.58).

Applying now (4.56), we obtain

$$
u_{0}(x, t)=\operatorname{cost}(t)+x, \quad u_{1}(x, t)=F_{2}^{-1}\left[G_{2}-I\right] u_{0}(x, t)=\frac{-x^{3}}{3 !},
$$

and in general we observe that

$$
u_{k+1}(x, t)=F_{2}^{-1}\left[G_{2}-I\right] u_{k}(x, t)=\frac{(-1)^{k+1} x^{2 k+3}}{(2 k+3) !}, \quad \forall k \geq 0 .
$$

Then (2.11) gives the ADM partial $x$-solution of problem (4.57) and (4.59) as

$$
u(x, t)=\cosh (t)+\sum_{k=0}^{\infty}(-1)^{k} \frac{x^{2 k+1}}{(2 k+1) !}=\cosh (t)+\sin (x), \quad(x, t) \in[0, \infty) \times \mathbb{R},
$$

as required; see (4.62)

Example 4.5. Take $\beta_{2}=1, \alpha_{0}=-2, f(x, t)=-2 \sin (x) \sin (t)$ in (4.54) to yield

$$
\frac{\partial^{2} u}{\partial t^{2}}(x, t)-2 u(x, t)=\frac{\partial^{2} u}{\partial x^{2}}(x, t)-2 \sin (x) \sin (t), \quad(x, t) \in \Omega,
$$


and consider the initial and boundary conditions

$$
\begin{array}{cl}
u(x, 0)=0=g_{0}(x), & \frac{\partial u}{\partial t}(x, 0)=\sin (x)=g_{1}(x), \quad x \in \mathbb{R} \\
u(0, t)=0=f_{0}(t), & \frac{\partial u}{\partial x}(0, t)=\sin (t)=f_{1}(t), \quad t \in \mathbb{R} .
\end{array}
$$

Calculating the first term in (4.55), we obtain

$$
u_{0}(x, t)=t \sin (x)+G_{2}^{-1}(-2 \sin (x) \sin (t))=-t \sin (x)+2 \sin (x) \sin (t) .
$$

As in Example 4.3, by observing that the starting term (4.69) can be decomposed into two parts, namely,

$$
u_{0}(x, t)=z_{1}(x, t)+z_{2}(x, t), \quad z_{1}(x, t)=\sin (x) \sin (t), \quad z_{2}(x, t)=-t \sin (x)+\sin (x) \sin (t)
$$

we use a slightly modified ADM instead of (4.55), namely, $u_{0}(x, t)=z_{1}(x, t)=\sin (x) \sin (t)$, $u_{1}(x, t)=-t \sin (x)+\sin (x) \sin (t)+G_{2}^{-1}\left(F_{2}+2 I\right) u_{0}(x, t)=0$, and in general $u_{k+1}(x, t)=$ $G_{2}^{-1}\left(F_{2}+2 I\right) u_{k}(x, t)=0$ for all $k \geq 0$. Then (2.11) gives the ADM partial $t$-solution

$$
u(x, t)=u_{0}(x, t)=\sin (x) \sin (t), \quad(x, t) \in \mathbb{R} \times[0, \infty),
$$

with only one term. It can easily be verified that (4.71) is the exact solution of (4.66) and (4.67). The solution (4.71) was previously obtained in [21] using the classical ADM based on (4.55) with the starting term (4.69), but the calculus employed in [21] is more complicated.

Calculating now the first term in (4.56), we obtain

$$
u_{0}(x, t)=x \sin (t)-F_{2}^{-1}(-2 \sin (x) \sin (t))=3 x \sin (t)-2 \sin (x) \sin (t) .
$$

As before, we decompose this term into two parts, namely,

$u_{0}(x, t)=z_{1}(x, t)+z_{2}(x, t), \quad z_{1}(x, t)=\sin (x) \sin (t), \quad z_{2}(x, t)=3 x \sin (t)-3 \sin (x) \sin (t)$,

and use a slightly modified ADM instead of (4.56), namely, $u_{0}(x, t)=z_{1}(x, t)=\sin (x) \sin (t)$, $u_{1}(x, t)=3 x \sin (t)-3 \sin (x) \sin (t)+F_{2}^{-1}\left(G_{2}-2 I\right) u_{0}(x, t)=0$, and in general $u_{k+1}(x, t)=$ $F_{2}^{-1}\left(G_{2}-2 I\right) u_{k}(x, t)=0$ for all $k \geq 0$. Again (2.11) gives the ADM partial $x$-solution of (4.66) and (4.68) in only one term $u(x, t)=u_{0}(x, t)=\sin (x) \sin (t)$, as required; see (4.71).

Note that if we take $\beta_{2}=-1$ in (4.54) then for $\alpha_{0}>0$ we obtain the two-dimensional Schrodinger (modified Helmholtz) equation, which was investigated using the ADM in [18], whilst for $\alpha_{0}<0$ we obtain the two-dimensional Helmholtz equation, which was investigated using the ADM in $[4,23]$. 
4.3.3. The linear dissipative wave equation. Consider the linear dissipative wave equation

$$
\alpha_{1} \frac{\partial u}{\partial t}(x, t)+\frac{\partial^{2} u}{\partial t^{2}}(x, t)=\beta_{2} \frac{\partial^{2} u}{\partial x^{2}}(x, t)+\beta_{1} \frac{\partial u}{\partial x}(x, t)+f(x, t), \quad(x, t) \in \Omega,
$$

which corresponds to the case $N=M=2, \alpha_{0}=0, \alpha_{2}=1$ in (1.1).

If (4.74) is solved subject to the initial conditions (4.38), then (2.9) gives

$$
\begin{gathered}
u_{0}(x, t)=g_{0}(x)+\operatorname{tg}_{1}(x)+G_{2}^{-1} f(x, t), \\
u_{k+1}(x, t)=G_{2}^{-1}\left[\beta_{2} F_{2}+\beta_{1} F_{1}-\alpha_{1} G_{1}\right] u_{k}(x, t), \quad k \geq 0,
\end{gathered}
$$

whilst if (4.74) is solved subject to the boundary conditions (4.16), then (2.10) gives

$$
\begin{gathered}
u_{0}(x, t)=f_{0}(t)+x f_{1}(t)-F_{2}^{-1}\left(\frac{f(x, t)}{\beta_{2}}\right), \\
u_{k+1}(x, t)=F_{2}^{-1}\left(\frac{1}{\beta_{2}} G_{2}+\frac{\alpha_{1}}{\beta_{2}} G_{1}-\frac{\beta_{1}}{\beta_{2}} F_{1}\right) u_{k}(x, t), \quad k \geq 0 .
\end{gathered}
$$

Example 4.6. Take $\beta_{2}=\alpha_{1}=\beta_{1}=1, f(x, t)=2(t-x)$ in (4.74) to yield

$$
\frac{\partial u}{\partial t}(x, t)+\frac{\partial^{2} u}{\partial t^{2}}(x, t)=\frac{\partial^{2} u}{\partial x^{2}}(x, t)+\frac{\partial u}{\partial x}(x, t)+2(t-x), \quad(x, t) \in \Omega,
$$

and consider the initial and boundary conditions

$$
\begin{array}{cl}
u(x, 0)=x^{2}=g_{0}(x), & \frac{\partial u}{\partial t}(x, 0)=0=g_{1}(x), \quad x \in \mathbb{R} \\
u(0, t)=t^{2}=f_{0}(t), & \frac{\partial u}{\partial x}(0, t)=0=f_{1}(t), \quad t \in \mathbb{R} .
\end{array}
$$

Calculating the first term in (4.75), we obtain

$$
u_{0}(x, t)=x^{2}+G_{2}^{-1}(2(t-x))=x^{2}+\frac{t^{3}}{3}-x t^{2},
$$

which can be decomposed into two parts, namely,

$$
u_{0}(x, t)=z_{1}(x, t)+z_{2}(x, t), \quad z_{1}(x, t)=x^{2}, \quad z_{2}(x, t)=\frac{t^{3}}{3}-x t^{2},
$$

and use the modified ADM to give

$$
\begin{gathered}
u_{0}(x, t)=z_{1}(x, t)=x^{2}, \quad u_{1}(x, t)=\frac{t^{3}}{3}-x t^{2}+G_{2}^{-1}\left[F_{2}+F_{1}-G_{1}\right] u_{0}(x, t)=t^{2}+\frac{t^{3}}{3}, \\
u_{2}(x, t)=G_{2}^{-1}\left[F_{2}+F_{1}-G_{1}\right] u_{1}(x, t)=\frac{t^{4}}{12}-\frac{t^{3}}{3}
\end{gathered}
$$


and so on. We observe then that (2.11) gives the ADM partial $t$-solution

$$
u(x, t)=u_{0}(x, t)+u_{1}(x, t)+u_{2}(x, t)+\cdots=x^{2}+t^{2} .
$$

It is easy to verify that (4.83) is the exact solution of (4.77) and (4.78). The solution (4.83) was previously obtained in [27] using the classical ADM based on (4.75) with the starting term (4.80), but the calculus in [27] is more complicated.

Calculating now the first term in (4.76), we obtain

$$
u_{0}(x, t)=t^{2}-F_{2}^{-1}(2(t-x))=t^{2}-t x^{2}+\frac{x^{3}}{3}
$$

As before, splitting $u_{0}$ into two parts and replacing everywhere $x$ with $t$ and $t$ with $x$ in the above equation, we obtain that the ADM partial $x$-solution of problem (4.77) and (4.79) is equal to the exact solution (4.83).

Note that if we take $\beta_{2}=-1$ in (4.74), we obtain the steady-state advection-diffusion equation.

4.4. The Korteweg-de Vries equation $(N=1, M=3)$. The linear Korteweg-de Vries $(\mathrm{KdV})$ equation

$$
\frac{\partial u}{\partial t}(x, t)=\beta_{3} \frac{\partial^{3} u}{\partial x^{3}}(x, t)+\beta_{1} \frac{\partial u}{\partial x}(x, t)+f(x, t), \quad(x, t) \in \Omega,
$$

governs long water waves, in water of relatively shallow depth, for very small amplitudes, see [46]. Equation (4.85) corresponds to the case $N=1, M=3, \alpha_{0}=\beta_{2}=0, \alpha_{1}=1$ in (1.1). When $\beta_{1}=0,(4.85)$ represents a third-order dispersive equation, see [44].

If (4.85) is solved subject to the initial condition (4.2), then (2.9) gives

$$
u_{0}(x, t)=g_{0}(x)+G_{1}^{-1} f(x, t), \quad u_{k+1}(x, t)=G_{1}^{-1}\left[\beta_{3} F_{3}+\beta_{1} F_{1}\right] u_{k}(x, t), \quad k \geq 0,
$$

whilst if (4.85) is solved subject to the lateral boundary conditions

$$
u(0, t)=f_{0}(f), \quad \frac{\partial u}{\partial x}(0, t)=f_{1}(t), \quad \frac{\partial^{2} u}{\partial x^{2}}(0, t)=f_{2}(t), \quad t \in \mathbb{R},
$$

then (2.10) gives

$$
\begin{aligned}
& u_{0}(x, t)=f_{0}(t)+x f_{1}(t)+\frac{x^{2}}{2} f_{2}(t)-F_{3}^{-1}\left(\frac{f(x, t)}{\beta_{3}}\right), \\
& u_{k+1}(x, t)=F_{3}^{-1}\left[\frac{1}{\beta_{3}} G_{1}-\frac{\beta_{1}}{\beta_{3}} F_{1}\right] u_{k}(x, t), \quad k \geq 0 .
\end{aligned}
$$

Example 4.7. Taking $\beta_{3}=\beta_{1}=-1, f=0$, then (4.85) becomes

$$
\frac{\partial u}{\partial t}(x, t)=-\frac{\partial^{3} u}{\partial x^{3}}(x, t)-\frac{\partial u}{\partial x}(x, t), \quad(x, t) \in \Omega,
$$


and consider the initial and boundary conditions

$$
\begin{gathered}
u(x, 0)=e^{-x}=g_{0}(x), \quad x \in \mathbb{R}, \\
u(0, t)=e^{2 t}=f_{0}(f), \quad \frac{\partial u}{\partial x}(0, t)=-e^{2 t}=f_{1}(t), \quad \frac{\partial^{2} u}{\partial x^{2}}(0, t)=e^{2 t}=f_{2}(t), \quad t \in \mathbb{R} .
\end{gathered}
$$

Applying (4.86), we obtain

$$
\begin{gathered}
u_{0}(x, t)=e^{-x}, \quad u_{1}(x, t)=G_{1}^{-1}\left[-F_{3}-F_{1}\right] e^{-x}=2 t e^{-x} \\
u_{2}(x, t)=G_{1}^{-1}\left[-F_{3}-F_{1}\right]\left(2 t e^{-x}\right)=2 t^{2} e^{-x}
\end{gathered}
$$

and in general we observe that $u_{k}(x, t)=\left((2 t)^{k} / k !\right) e^{-x}$ for all $k \geq 0$. Then (2.11) gives the ADM partial $t$-solution (see [28])

$$
u(x, t)=e^{-x} \sum_{k=0}^{\infty} \frac{(2 t)^{k}}{k !}=e^{2 t-x}, \quad(x, t) \in \mathbb{R} \times[0, \infty),
$$

which can be verified through substitution to be the exact solution of (4.89) and (4.90).

Applying now (4.88), we obtain

$$
\begin{gathered}
u_{0}(x, t)=e^{2 t}\left(1-x+\frac{x^{2}}{2}\right), \quad u_{1}(x, t)=F_{3}^{-1}\left(-G_{1}-F_{1}\right) u_{0}(x, t)=e^{2 t}\left(-\frac{x^{3}}{3 !}+\frac{x^{4}}{4 !}-\frac{2 x^{5}}{5 !}\right) \\
u_{2}(x, t)=F_{3}^{-1}\left(-G_{1}-F_{1}\right) u_{1}(x, t)=e^{2 t}\left(\frac{x^{5}}{5 !}+\frac{x^{6}}{6 !}+\frac{4 x^{8}}{8 !}\right) \\
u_{3}(x, t)=F_{3}^{-1}\left(-G_{1}-F_{1}\right) u_{2}(x, t)=e^{2 t}\left(-\frac{x^{7}}{7 !}-\frac{3 x^{8}}{8 !}-\frac{4 x^{1} 0}{10 !}-\frac{8 x^{1} 1}{11 !}\right)
\end{gathered}
$$

and so forth and in general we observe that based on (2.11), the ADM partial $x$-solution of problem (4.89) and (4.91) is given by

$$
\begin{aligned}
u(x, t) & =u_{0}+u_{1}+u_{2}+u_{3}+\cdots \\
& =e^{2 t}\left(1-x+\frac{x^{2}}{2 !}-\frac{x^{3}}{3 !}+\frac{x^{4}}{4 !}-\frac{x^{5}}{5 !}+\frac{x^{6}}{6 !}-\frac{x^{7}}{7 !}+\frac{x^{8}}{8 !}-\cdots\right) \\
& =e^{2 t-x}, \quad(x, t) \in[0, \infty) \times \mathbb{R},
\end{aligned}
$$

as required; see also (4.93).

Example 4.8. Take $\beta_{1}=0, \beta_{3}=1$ and $f(x, t)=2 e^{t-x}$ in (4.85) to obtain the linear thirdorder dispersive, inhomogeneous equation

$$
\frac{\partial u}{\partial t}(x, t)=\frac{\partial^{3} u}{\partial x^{3}}(x, t)+2 e^{t-x}, \quad(x, t) \in \Omega
$$


18 The decomposition method for linear PDEs

and consider the initial and boundary conditions

$$
\begin{gathered}
u(x, 0)=1+e^{-x}=g_{0}(x), \quad x \in \mathbb{R}, \\
u(0, t)=1+e^{t}=f_{0}(f), \quad \frac{\partial u}{\partial x}(0, t)=-e^{t}=f_{1}(t), \quad \frac{\partial^{2} u}{\partial x^{2}}(0, t)=e^{t}=f_{2}(t), \quad t \in \mathbb{R} .
\end{gathered}
$$

If (4.96) is solved subject to the initial condition (4.97), then (2.9) gives

$$
\begin{gathered}
u_{0}(x, t)=g_{0}(x)+G_{1}^{-1} f(x, t)=1-e^{-x}+2 e^{t-x}, \\
u_{1}(x, t)=G_{1}^{-1} F_{3} u_{0}(x, t)=(t+2) e^{-x}-2 e^{t-x}, \\
u_{2}(x, t)=G_{1}^{-1} F_{3} u_{1}(x, t)=-\left(\frac{t^{2}}{2 !}+2 t+2\right) e^{-x}+2 e^{t-x}, \\
u_{3}(x, t)=G_{1}^{-1} F_{3} u_{2}(x, t)=\left(\frac{t^{3}}{3 !}+t^{2}+2 t+2\right) e^{-x}-2 e^{t-x},
\end{gathered}
$$

and so on. It is clear that the self-cancelling "noise" terms appear between various components, and keeping the noncancelled terms and using (2.11) lead immediately to the ADM partial $t$-solution (see [28])

$$
\begin{aligned}
u(x, t) & =u_{0}+u_{1}+u_{2}+u_{3}+\cdots \\
& =1+e^{-x}\left(1+t+\frac{t^{2}}{2 !}+\frac{t^{3}}{3 !}+\cdots\right) \\
& =1+e^{t-x}, \quad(x, t) \in \mathbb{R} \times[0, \infty),
\end{aligned}
$$

which can be verified through substitution to be the exact solution of problem (4.96) and (4.97).

If now (4.96) is solved subject to the boundary conditions (4.98), then (2.10) gives

$$
\begin{aligned}
& u_{0}(x, t)=f_{0}(t)+x f_{1}(t)+\frac{x^{2}}{2} f_{2}(t)-F_{3}^{-1} f(x, t)=2 e^{t-x}+1+e^{t}\left(1-x+\frac{x^{2}}{2}\right), \\
& u_{1}(x, t)=F_{3}^{-1} G_{1} u_{0}(x, t)=-2 e^{t-x}+2 e^{t}\left(1-x+\frac{x^{2}}{2}\right)+e^{t}\left(\frac{x^{3}}{3 !}-\frac{x^{4}}{4 !}+\frac{x^{5}}{5 !}\right), \\
& u_{2}(x, t)=F_{3}^{-1} G_{1} u_{1}(x, t)=2 e^{t-x}-2 e^{t}\left(1-x+\frac{x^{2}}{2}\right)+2 e^{t}\left(\frac{x^{3}}{3 !}-\frac{x^{4}}{4 !}+\frac{x^{5}}{5 !}\right) \\
& +e^{t}\left(\frac{x^{6}}{6 !}-\frac{x^{7}}{7 !}+\frac{x^{8}}{8 !}\right) \\
& u_{3}(x, t)=F_{3}^{-1} G_{1} u_{2}(x, t)=-2 e^{t-x}+2 e^{t}\left(1-x+\frac{x^{2}}{2}\right)-2 e^{t}\left(\frac{x^{3}}{3 !}-\frac{x^{4}}{4 !}+\frac{x^{5}}{5 !}\right) \\
& +2 e^{t}\left(\frac{x^{6}}{6 !}-\frac{x^{7}}{7 !}+\frac{x^{8}}{8 !}\right)+e^{t}\left(\frac{x^{9}}{9 !}-\frac{x^{10}}{10 !}+\frac{x^{11}}{11 !}\right) \text {, }
\end{aligned}
$$


and so on. In the above, one obtains self-cancelling "noise" terms appearing between various components of $u_{0}, u_{1}, u_{2}, u_{3}, \ldots$, and keeping the noncancelled terms, and using (2.11) lead to the ADM partial $x$-solution of problem (4.96) and (4.98) as

$$
\begin{aligned}
u(x, t) & =u_{0}+u_{1}+u_{2}+u_{3}+\cdots \\
& =1+e^{t}\left(1-x+\frac{x^{2}}{2 !}-\frac{x^{3}}{3 !}+\cdots\right) \\
& =1+e^{t-x}, \quad(x, t) \in[0, \infty) \times \mathbb{R},
\end{aligned}
$$

as required; see also (4.100).

It is worth noting that noise terms between components of the decomposition series will be cancelled, and the sum of these "noise" terms will vanish in the limit; see $[10,41]$.

Alternatively, since the starting term $u_{0}$ in (4.101) can be decomposed into two parts, namely,

$$
u_{0}(x, t)=z_{1}(x, t)+z_{2}(x, t), \quad z_{1}(x, t)=1+e^{t-x}, \quad z_{2}(x, t)=e^{t-x}+e^{t}\left(1-x+\frac{x^{2}}{2}\right)
$$

then a slightly modified recursive algorithm can be used (see [43]), namely,

$$
\begin{gathered}
u_{0}(x, t)=z_{1}(x, t)=1+e^{t-x}, \\
u_{1}(x, t)=z_{2}(x, t)+F_{3}^{-1} G_{1} u_{0}(x, t)=e^{t-x}+e^{t}\left(1-x+\frac{x^{2}}{2}\right)+F_{3}^{-1} G_{1}\left(1+e^{t-x}\right)=0,
\end{gathered}
$$

and in general $u_{k+1}(x, t)=F_{3}^{-1} G_{1} u_{k}(x, t)=0$ for all $k \geq 1$. Then the exact solution (4.100) of (4.96) and (4.98) is obtained with only one term $u=u_{0}$ in the decomposition series (2.11).

Example 4.9. We consider the example tested in [26] obtained by taking $\beta_{1}=\beta_{3}=-1$ and $f(x, t)=1+(1+t) e^{x}+e^{2 x}$, in which case (4.85) becomes

$$
\frac{\partial u}{\partial t}(x, t)=-\frac{\partial^{3} u}{\partial x^{3}}(x, t)-\frac{\partial u}{\partial x}(x, t)+1+(1+t) e^{x}+e^{2 x}, \quad(x, t) \in \Omega .
$$

At this stage, we note that the "exact" solution $u(x, t)=e^{x}+t$ obtained in [26] is incorrect since it does not satisfy (4.105). We remedy this mistake by taking the exact solution of (4.105) as

$$
u(x, t)=t\left(1+\frac{e^{x}}{2}\right)+\frac{e^{x}}{4}+\frac{e^{2 x}}{10}
$$


which generates the initial and boundary conditions

$$
\begin{array}{cc}
u(x, 0)=\frac{e^{x}}{4}+\frac{e^{2 x}}{10}=g_{0}(x), \quad x \in \mathbb{R}, & \\
u(0, t)=\frac{3 t}{2}+\frac{7}{20}=f_{0}(t), \quad t \in \mathbb{R}, \\
\frac{\partial u}{\partial x}(0, t)=\frac{t}{2}+\frac{9}{20}=f_{1}(t), \quad t \in \mathbb{R}, \\
\frac{\partial^{2} u}{\partial x^{2}}(0, t)=\frac{t}{2}+\frac{13}{20}=f_{2}(t), \quad t \in \mathbb{R} .
\end{array}
$$

If (4.105) is solved subject to the initial condition (4.107), then (4.86) gives

$$
u_{0}(x, t)=\frac{e^{x}}{4}+\frac{e^{2 x}}{10}+t+\left(t+\frac{t^{2}}{2}\right) e^{x}+t e^{2 x}
$$

which can be decomposed into two parts, namely,

$$
u_{0}(x, t)=z_{1}(x, t)+z_{2}(x, t), \quad z_{1}(x, t)=\frac{e^{x}}{4}+\frac{e^{2 x}}{10}+t, \quad z_{2}(x, t)=\left(t+\frac{t^{2}}{2}\right) e^{x}+t e^{2 x},
$$

and use the modified recursive algorithm

$$
\begin{aligned}
& u_{0}(x, t)=z_{1}(x, t)=\frac{e^{x}}{4}+\frac{e^{2 x}}{10}+t, \\
& u_{1}(x, t)=z_{2}(x, t)+G_{1}^{-1}\left[-F_{3}-F_{1}\right] u_{0}(x, t)=\frac{t e^{x}}{2}+\frac{t^{2} e^{x}}{2}, \\
& u_{2}(x, t)=G_{1}^{-1}\left[-F_{3}-F_{1}\right] u_{1}(x, t)=-\frac{t^{2} e^{x}}{2}-\frac{t^{3} e^{x}}{3}, \\
& u_{3}(x, t)=G_{1}^{-1}\left[-F_{3}-F_{1}\right] u_{2}(x, t)=\frac{t^{3} e^{x}}{3}+\frac{t^{4} e^{x}}{6},
\end{aligned}
$$

and so on. We observe that terms in the second term of $u_{k}$ cancel with the first term of $u_{k+1}$ for $k \geq 1$. Then (2.11) gives the exact solution (4.106), as required.

At this stage, we note that the decomposition of $u_{0}$ in (4.110) is not unique, and for example, if one selects a better decomposition such as

$$
u_{0}(x, t)=z_{1}(x, t)+z_{2}(x, t), \quad z_{1}(x, t)=\frac{e^{x}}{4}+\frac{e^{2 x}}{10}+t+\frac{t e^{x}}{2}, \quad z_{2}(x, t)=\frac{\left(t+t^{2}\right)}{2} e^{x}+t e^{2 x},
$$

then the exact solution (4.106) is obtained with only one term $u=u_{0}$ in the series (2.11). Some idea about appropriate choices in the decomposition of $u_{0}$ have been recently discussed by Lesnic and Elliott [33] who proposed a two-step ADM in which various parts of $u_{0}$ are tested if they satisfy the governing equation and/or the initial and/or boundary conditions. 
If (4.105) is now solved subject to (4.108), then (4.88) gives

$$
\begin{aligned}
u_{0}(x, t)= & \frac{t}{2}\left(3+x+\frac{x^{2}}{2 !}\right)+\frac{1}{20}\left(7+9 x+\frac{13 x^{2}}{2 !}\right)+F_{3}^{-1}\left(1+(1+t) e^{x}+e^{2 x}\right) \\
= & \frac{e^{2 x}}{8}+(1+t) e^{x}+\frac{x^{3}}{3 !}-\frac{17 x^{2}+32 x+31}{40}+\frac{t}{2}\left(1-x-\frac{x}{2}\right) \\
u_{1}(x, t)= & F_{3}^{-1}\left[-G_{1}-F_{1}\right] u_{0}(x, t) \\
= & -\frac{e^{2 x}}{32}-(2+t) e^{x}+\frac{975+990 x+510 x^{2}+24 x^{3}+27 x^{4}-2 x^{5}}{480} \\
& +t\left(1+x+\frac{x^{2}}{2}+\frac{x^{3}}{12}+\frac{x^{4}}{48}\right) .
\end{aligned}
$$

As the calculus becomes complicated, one can use MAPLE, or alternatively, one can decompose the initial term $u_{0}$ given by (4.113) into two parts, namely,

$$
\begin{array}{r}
u_{0}(x, t)=z_{1}(x, t)+z_{2}(x, t), \quad z_{1}(x, t)=\frac{e^{2 x}}{10}+\left(t+\frac{1}{2}\right) \frac{e^{x}}{2}+t \\
z_{2}(x, t)=\frac{x^{3}}{3 !}-\frac{17 x^{2}+32 x+31}{40}-\frac{t}{2}\left(1+x+\frac{x^{2}}{2}\right)+\frac{e^{2 x}}{40}+\frac{e^{x}}{2}\left(t+\frac{3}{2}\right),
\end{array}
$$

and use the modified recursive algorithm

$$
\begin{gathered}
u_{0}(x, t)=z_{1}(x, t)=\frac{e^{2 x}}{10}+\left(t+\frac{1}{2}\right) \frac{e^{x}}{2}+t, \quad u_{1}(x, t)=z_{2}(x, t)+F_{3}^{-1}\left[-G_{1}-F_{1}\right] u_{0}(x, t)=0, \\
u_{k+1}(x, t)=F_{3}^{-1}\left[-G_{1}-F_{1}\right] u_{k}(x, t)=0, \quad \forall k \geq 1,
\end{gathered}
$$

to obtain the exact solution (4.106) of problem (4.105) and (4.108) with only one term $u=u_{0}$ in the series (2.11).

Since the case $N=1, M=4$ of (1.1) is not a model of any well-known physical situation, it is not considered here, although one may think of it as a fourth-order diffusion process, see [25].

4.5. The Euler-Bernoulli equation $(N=2, M=4)$. The Euler-Bernoulli equation

$$
-\frac{\partial^{2} u}{\partial t^{2}}(x, t)=\beta_{4} \frac{\partial^{4} u}{\partial x^{4}}(x, t)+f(x, t), \quad(x, t) \in \Omega,
$$

governs the deflection of an elastic beam under the action of a load $f(x, t)$. In (4.116), the solution $u$ represents the deflection of the beam and $\beta_{4}>0$ is its flexural rigidity. Equation (4.116) corresponds to the case $N=2, M=4, \alpha_{0}=\alpha_{1}=\beta_{1}=\beta_{2}=\beta_{3}=0, \alpha_{2}=-1$ in (1.1).

If (4.116) is solved subject to the initial conditions (4.38), then (2.9) gives

$$
u_{0}(x, t)=g_{0}(x)+\operatorname{tg}_{1}(x)-G_{2}^{-1} f(x, t), \quad u_{k+1}(x, t)=G_{2}^{-1}\left[-\beta_{4} F_{4}\right] u_{k}(x, t), \quad k \geq 0,
$$


whilst if (4.116) is solved subject to the lateral boundary conditions

$$
u(0, t)=f_{0}(t), \quad \frac{\partial u}{\partial x}(0, t)=f_{1}(t), \quad \frac{\partial^{2} u}{\partial x^{2}}(0, t)=f_{2}(t), \quad \frac{\partial^{3} u}{\partial x^{3}}(0, t)=f_{3}(t), \quad t \in \mathbb{R},
$$

then (2.10) gives

$$
\begin{gathered}
u_{0}(x, t)=f_{0}(t)+x f_{1}(t)+\frac{x^{2}}{2 !} f_{2}(t)+\frac{x^{3}}{3 !} f_{3}(t)-F_{4}^{-1}\left(\frac{f(x, t)}{\beta_{4}}\right), \\
u_{k+1}(x, t)=F_{4}^{-1}\left(-\frac{1}{\beta_{4}} G_{2}\right) u_{k}(x, t), \quad k \geq 0 .
\end{gathered}
$$

When $f(x, t)=0$, (4.116) becomes

$$
-\frac{\partial^{2} u}{\partial t^{2}}(x, t)=\beta_{4} \frac{\partial^{4} u}{\partial x^{4}}(x, t), \quad(x, t) \in \Omega,
$$

which is a particular case of (3.1) with $N=2, M=4$, and $\alpha_{2}=-1$. Then (3.4) gives the ADM partial $t$-solution of problem (4.38) and (4.120) as

$$
u(x, t)=\sum_{k=0}^{\infty}\left(-\beta_{4}\right)^{k}\left[g_{0}^{(4 k)}(x) \frac{t^{2 k}}{(2 k) !}+g_{1}^{(4 k)}(x) \frac{t^{2 k+1}}{(2 k+1) !}\right], \quad(x, t) \in \mathbb{R} \times[0, \infty),
$$

whilst (3.5) gives the ADM partial $x$-solution of problem (4.118) and (4.120) as

$$
u(x, t)=\sum_{k=0}^{\infty}\left(-\frac{1}{\beta_{4}}\right)^{k} \sum_{l=0}^{3} f_{l}^{(2 k)}(t) \frac{x^{4 k+l}}{(4 k+l) !}, \quad(x, t) \in[0, \infty) \times \mathbb{R} .
$$

Example 4.10. Take $\beta_{4}=1$ and $f(x, t)=-x t-t^{2}$ in (4.116) to yield

$$
-\frac{\partial^{2} u}{\partial t^{2}}(x, t)=\beta_{4} \frac{\partial^{4} u}{\partial x^{4}}(x, t)-x t-t^{2}, \quad(x, t) \in \Omega,
$$

and consider the initial and boundary conditions

$$
\begin{gathered}
u(x, 0)=0=g_{0}(x), \quad \frac{\partial u}{\partial t}(x, 0)=\frac{x^{5}}{5 !}=g_{1}(x), \quad x \in \mathbb{R}, \\
u(0, t)=\frac{t^{4}}{12}=f_{0}(t), \quad \frac{\partial^{l} u}{\partial x^{l}}=0=f_{l}(t), \quad l=1,2,3, t \in \mathbb{R} .
\end{gathered}
$$

If (4.123) is solved subject to the initial conditions (4.124), then (4.117) gives

$$
\begin{gathered}
u_{0}(x, t)=\frac{x^{5} t}{5 !}+G_{2}^{-1}\left(x t+t^{2}\right)=\frac{x^{5} t}{5 !}+\frac{t^{4}}{12}+\frac{x t^{3}}{6}, \\
u_{1}(x, t)=G_{2}^{-1}\left(-F_{4}\right) u_{0}(x, t)=-\frac{x t^{3}}{6}, \quad u_{2}(x, t)=G_{2}^{-1}\left(-F_{4}\right) u_{1}(x, t)=0,
\end{gathered}
$$


and in general $u_{k+1}(x, t)=G_{2}^{-1}\left(-F_{4}\right) u_{k}(x, t)=0$ for all $k \geq 1$. Thus the ADM partial $t$-solution, via (2.11), gives the exact solution $u=u_{0}+u_{1}=t x^{5} / 5 !+t^{4} / 12$ of problem (4.123) and (4.124) in only two terms.

If (4.123) is now solved subject to the boundary conditions (4.125), then (4.119) gives

$$
\begin{gathered}
u_{0}(x, t)=\frac{t^{4}}{12}+F_{4}^{-1}\left(x t+t^{2}\right)=\frac{x^{5} t}{5 !}+\frac{t^{4}}{12}+\frac{t^{2} x^{4}}{4 !}, \\
u_{1}(x, t)=F_{4}^{-1}\left(-G_{2}\right) u_{0}(x, t)=-\frac{t^{2} x^{4}}{4 !}-\frac{2 x^{8}}{8 !}, \\
u_{2}(x, t)=F_{4}^{-1}\left(-G_{2}\right) u_{1}(x, t)=\frac{2 x^{8}}{8 !}, \quad u_{3}(x, t)=F_{4}^{-1}\left(-G_{2}\right) u_{2}(x, t)=0,
\end{gathered}
$$

and in general $u_{k+1}(x, t)=F_{4}^{-1}\left(-G_{2}\right) u_{k}(x, t)=0$ for all $k \geq 2$. Thus the ADM partial $x$ solution, via (2.11), gives the exact solution $u=u_{0}+u_{1}+u_{2}=t x^{5} / 5 !+t^{4} / 12$ of problem (4.123) and (4.125) in only three terms.

Since the case $N=1, M=5$ of (1.1) is not a model of any well-known physical situation, it is not considered here, although one may think of it as a linear fifth-order KdV equation, see [30]. Finally, we mention that the case $N=1, M=6$ of (1.1) may be thought of as a model equation for linear seismic waves, see [7]. The ADM described in this study can also be applied to these equations.

\section{Extension to systems of linear PDEs}

The ADM can easily be extended to systems of linear PDEs of the form

$$
\begin{aligned}
& \sum_{n=0}^{N} \alpha_{n}(x, t) \frac{\partial^{n} u}{\partial t^{n}}(x, t)=\beta_{0}(x, t) v(x, t)+\sum_{m=1}^{M} \beta_{m}(x, t) \frac{\partial^{m} u}{\partial x^{m}}(x, t)+f(x, t), \quad(x, t) \in \Omega, \\
& \sum_{n=0}^{N_{1}} \gamma_{n}(x, t) \frac{\partial^{n} v}{\partial t^{n}}(x, t)=\delta_{0}(x, t) u(x, t)+\sum_{m=1}^{M_{1}} \delta_{m}(x, t) \frac{\partial^{m} v}{\partial x^{m}}(x, t)+g(x, t), \quad(x, t) \in \Omega,
\end{aligned}
$$

where $f, g,\left(\alpha_{i}\right)_{i=\overline{0, N}},\left(\beta_{i}\right)_{i=\overline{0, M}},\left(\gamma_{i}\right)_{i=\overline{0, N_{1}}},\left(\delta_{i}\right)_{i=\overline{0, M_{1}}}$ are given coefficients, $\alpha_{N} \neq 0, \beta_{M} \neq 0$, $\gamma_{N_{1}} \neq 0, \delta_{M_{1}} \neq 0$, and $N, M, N_{1}, M_{1}$ are positive integers. The system of PDEs (5.1) has then to be solved subject to the initial conditions (1.2) and

$$
\frac{\partial^{n} v}{\partial t^{n}}(x, 0)=h_{n}(x), \quad n=\overline{0,\left(N_{1}-1\right)}, x \in \mathbb{R}
$$

or to the lateral boundary conditions (1.3) and

$$
\frac{\partial^{m} v}{\partial x^{m}}(0, t)=i_{m}(t), \quad m=\overline{0,\left(M_{1}-1\right)}, t \in \mathbb{R}
$$

Then, similarly as in (2.9) and (2.10), the ADM partial $t$-solution of problem (1.2), (5.1), and (5.2), and the ADM partial $x$-solution of problem (1.3), (5.1), and (5.3) will be 
given by the decomposition series (2.11), where the components of the series are calculated recursively from the following relationships:

$$
\begin{aligned}
u_{0}(x, t)= & \sum_{l=0}^{N-1} \frac{t^{l}}{l !} g_{l}(x)+G_{N}^{-1}\left(\frac{f(x, t)}{\alpha_{N}(x, t)}\right), \quad v_{0}(x, t)=\sum_{l=0}^{N_{1}-1} \frac{t^{l}}{l !} h_{l}(x)+G_{N_{1}}^{-1}\left(\frac{g(x, t)}{\gamma_{N_{1}}(x, t)}\right), \\
u_{k+1}(x, t)= & {\left[\sum_{m=1}^{M} G_{N}^{-1}\left(\frac{\beta_{m}}{\alpha_{N}} F_{m}\right)-\sum_{n=0}^{N-1} G_{N}^{-1}\left(\frac{\alpha_{n}}{\alpha_{N}} G_{n}\right)\right] u_{k}(x, t)+G_{N}^{-1}\left(\frac{\beta_{0}}{\alpha_{N}} v_{k}(x, t)\right), \quad k \geq 0, } \\
v_{k+1}(x, t)= & {\left[\sum_{m=1}^{M_{1}} G_{N_{1}}^{-1}\left(\frac{\delta_{m}}{\gamma_{N_{1}}} F_{m}\right)-\sum_{n=0}^{N_{1}-1} G_{N_{1}}^{-1}\left(\frac{\gamma_{n}}{\gamma_{N_{1}}} G_{n}\right)\right] v_{k}(x, t)+G_{N_{1}}^{-1}\left(\frac{\delta_{0}}{\gamma_{N_{1}}} u_{k}(x, t)\right), \quad k \geq 0, } \\
u_{0}(x, t)= & \sum_{l=0}^{M-1} \frac{x^{l}}{l !} f_{l}(t)-F_{M}^{-1}\left(\frac{f(x, t)}{\beta_{M}(x, t)}\right), \quad v_{0}(x, t)=\sum_{l=0}^{M_{1}-1} \frac{x^{l}}{l !} i_{l}(t)-F_{M_{1}}^{-1}\left(\frac{g(x, t)}{\delta_{M_{1}}(x, t)}\right), \\
u_{k+1}(x, t)= & {\left[\sum_{n=0}^{N} F_{M}^{-1}\left(\frac{\alpha_{n}}{\beta_{M}} G_{n}\right)-\sum_{m=1}^{M-1} F_{M}^{-1}\left(\frac{\beta_{m}}{\beta_{M}} F_{m}\right)\right] u_{k}(x, t)-F_{M}^{-1}\left(\frac{\beta_{0}}{\beta_{M}} v_{k}(x, t)\right), \quad k \geq 0, } \\
v_{k+1}(x, t)= & {\left[\sum_{n=0}^{N_{1}} F_{M_{1}}^{-1}\left(\frac{\gamma_{n}}{\delta_{M_{1}}} G_{n}\right)-\sum_{m=1}^{M_{1}-1} F_{M_{1}}^{-1}\left(\frac{\delta_{m}}{\delta_{M_{1}}} F_{m}\right)\right] v_{k}(x, t)-F_{M_{1}}^{-1}\left(\frac{\delta_{0}}{\delta_{M_{1}}} u_{k}(x, t)\right), \quad k \geq 0, }
\end{aligned}
$$

respectively.

\section{Extension to nonlinear PDEs}

The ADM can also be extended to solving initial or boundary value problems for nonlinear, one-dimensional, time-dependent PDEs of the form

$$
\begin{aligned}
\sum_{n=1}^{N} \alpha_{n} & \frac{\partial^{n} u}{\partial t^{n}}(x, t)+A\left(u, \partial_{t} u, \ldots, \partial_{t}^{N-1} u\right) \\
& =\sum_{m=1}^{M} \beta_{m} \frac{\partial^{m} u}{\partial x^{m}}(x, t)+B\left(\partial_{x} u, \ldots, \partial_{x}^{M-1} u\right)+f(x, t), \quad(x, t) \in \Omega,
\end{aligned}
$$

where $\partial_{t}^{n} u=\partial^{n} u / \partial t^{n}$ for $n=\overline{0,(N-1)}$, and $\partial_{x}^{m} u=\partial^{m} u / \partial x^{m}$ for $m=\overline{1,(M-1)}$. Equation (6.1) has to be solved subject to the initial conditions (1.2), or to the lateral boundary conditions (1.3). Then, similarly as in (2.9) and (2.10), the ADM partial $t$-solution of problem (1.2) and (6.1), and the ADM partial $x$-solution of problem (1.3) and (6.1) will be given by the decomposition series (2.11), where the components of the series are 
calculated recursively from the following relationships:

$$
\begin{gathered}
u_{0}(x, t)=\sum_{l=0}^{N-1} \frac{t^{l}}{l !} g_{l}(x)+G_{N}^{-1}\left(\frac{f(x, t)}{\alpha_{N}(x, t)}\right), \\
u_{k+1}(x, t)=\left[\sum_{m=1}^{M} G_{N}^{-1}\left(\frac{\beta_{m}}{\alpha_{N}} F_{m}\right)-\sum_{n=1}^{N-1} G_{N}^{-1}\left(\frac{\alpha_{n}}{\alpha_{N}} G_{n}\right)\right] u_{k}(x, t)+G_{N}^{-1}\left(B_{k}-A_{k}\right), \quad k \geq 0, \\
u_{0}(x, t)=\sum_{l=0}^{M-1} \frac{x^{l}}{l !} f_{l}(t)-F_{M}^{-1}\left(\frac{f(x, t)}{\beta_{M}(x, t)}\right), \\
u_{k+1}(x, t)=\left[\sum_{n=1}^{M} F_{M}^{-1}\left(\frac{\alpha_{n}}{\beta_{M}} G_{n}\right)-\sum_{m=1}^{M-1} F_{M}^{-1}\left(\frac{\beta_{m}}{\beta_{M}} F_{m}\right)\right] u_{k}(x, t)+F_{M}^{-1}\left(A_{k}-B_{k}\right), \quad k \geq 0,
\end{gathered}
$$

respectively. In (6.2) and (6.3), $A_{k}$ and $B_{k}$ are called the Adomian polynomials. These polynomials can be calculated for all forms of analytical nonlinearities, according to specific algorithms given, for example, in $[6,37]$ as

$$
\begin{gathered}
A_{k}=\frac{1}{k !}\left[\frac{d^{k}}{d \lambda^{k}} A\left(\sum_{j=0}^{k} \lambda^{j} G_{0} u_{j}, \ldots, \sum_{j=0}^{k} \lambda^{j} G_{N-1} u_{j}\right)\right]_{\lambda=0}, \quad k \geq 0, \\
B_{k}=\frac{1}{k !}\left[\frac{d^{k}}{d \lambda^{k}} B\left(\sum_{j=0}^{k} \lambda^{j} F_{1} u_{j}, \ldots, \sum_{j=0}^{k} \lambda^{j} F_{M-1} u_{j}\right)\right]_{\lambda=0}, \quad k \geq 0 .
\end{gathered}
$$

For example, if $A(u)=u^{2}$, then $A_{k}=\sum_{l=0}^{k} u_{l} u_{l-k}$.

6.1. An application to a nonlinear PDE. Let us investigate the advection equation with a nonlinear term considered in [11] of the form

$$
\frac{\partial u}{\partial t}(x, t)=-\frac{\partial u}{\partial x}(x, t)-u^{2}(x, t), \quad(x, t) \in \Omega,
$$

which corresponds to the case $N=M=1, \alpha_{1}=1, \beta_{1}=-1, f=0, A(u)=u^{2}$ in (6.1).

If (6.6) is solved subject to the initial condition

$$
u(x, 0)=\frac{1}{2 x}=g_{0}(x), \quad x \in \mathbb{R}-\{0\},
$$

then on using the ADM given by (6.2) and (6.4), we obtain

$$
\begin{gathered}
u_{0}(x, t)=\frac{1}{2 x}, \quad A_{0}=u_{0}^{2}=\frac{1}{4 x^{2}}, \\
u_{1}(x, t)=-G_{1}^{-1} F_{1} u_{0}-G_{1}^{-1}\left(A_{0}\right)=\frac{t}{2 x^{2}}-\frac{t}{4 x^{2}}=\frac{t}{4 x^{2}}, \quad A_{1}=2 u_{0} u_{1}=\frac{t}{4 x^{3}}, \\
u_{2}(x, t)=-G_{1}^{-1} F_{1} u_{1}-G_{1}^{-1}\left(A_{1}\right)=\frac{t^{2}}{4 x^{3}}-\frac{t^{2}}{8 x^{3}}=\frac{t^{2}}{8 x^{3}},
\end{gathered}
$$


and in general one obtains $u_{k}(x, t)=t^{k} /(2 x)^{k+1}$ for $k \geq 0$. Then using (2.11), we obtain the ADM partial $t$-solution of problem (6.6) and (6.7) as given by

$$
u(x, t)=\sum_{k=0}^{\infty} \frac{t^{k}}{(2 x)^{k+1}}, \quad(x, t) \in(\mathbb{R}-\{0\}) \times[0, \infty),
$$

which, for $0 \leq t / 2|x|<1$, converges to the exact solution $u(x, t)=1 /(2 x-t)$.

If (6.6) is now solved subject to the boundary condition

$$
u(0, t)=-\frac{1}{t}, \quad t \in \mathbb{R}-\{0\},
$$

then on using the ADM given by (6.3) and (6.4), we obtain

$$
\begin{gathered}
u_{0}(x, t)=-\frac{1}{t}, \quad A_{0}=u_{0}^{2}=\frac{1}{t^{2}} \\
u_{1}(x, t)=-F_{1}^{-1} G_{1} u_{0}-F_{1}^{-1}\left(A_{0}\right)=-\frac{2 x}{t^{2}}, \quad A_{1}=2 u_{0} u_{1}=\frac{4 x}{t^{3}} \\
u_{2}(x, t)=-F_{1}^{-1} G_{1} u_{1}-F_{1}^{-1}\left(A_{1}\right)=-\frac{4 x^{2}}{t^{3}}, \quad A_{2}=2 u_{0} u_{2}+u_{1}^{2}=\frac{12 x^{2}}{t^{4}} \\
u_{3}(x, t)=-F_{1}^{-1} G_{1} u_{2}-F_{2}^{-1}\left(A_{1}\right)=-\frac{8 x^{3}}{t^{4}}
\end{gathered}
$$

and in general one obtains $u_{k}(x, t)=-(2 x)^{k} / t^{k+1}$ for $k \geq 0$. Then using (2.11), we obtain the ADM partial $x$-solution of problem (6.6) and (6.10) as given by

$$
u(x, t)=-\sum_{k=0}^{\infty} \frac{(2 x)^{k}}{t^{k+1}}, \quad(x, t) \in[0, \infty) \times(\mathbb{R}-\{0\}),
$$

which, for $0 \leq 2 x /|t|<1$, converges to the exact solution $u(x, t)=1 /(2 x-t)$.

It can be seen that the series (6.9) and (6.12) are quite different since they have different domains of convergence. Thus, the ADM partial $t$-solution (6.9) and partial $x$-solution (6.1) are not generally equivalent, but rather formally equivalent. In this sense, the equivalence of the ADM partial solutions considered in $[9,31,40]$ should be understood .

More physical examples of nonlinear PDEs will be investigated in a future work.

\section{Conclusions}

In this study, the ADM has been reviewed for solving initial or lateral boundary value problems for linear, one-dimensional, time-dependent PDEs given by (1.1). Furthermore, if the PDEs are homogeneous and have constant coefficients, see, for example, (3.1), (4.8), (4.18), and (4.24), then analytical solutions have been derived. Otherwise, the ADM gives the solution in the form of a series which in most cases is rapidly convergent, if slight modifications are also implemented, such as the phenomenon of cancelling "noise" terms, and the splitting of the initial term into two appropriate parts. 


\section{References}

[1] G. Adomian, A new approach to nonlinear partial differential equations, Journal of Mathematical Analysis and Applications 102 (1984), no. 2, 420-434.

[2] A A new approach to the heat equation - an application of the decomposition method, Journal of Mathematical Analysis and Applications 113 (1986), no. 1, 202-209.

[3] __ Modification of the decomposition approach to the heat equation, Journal of Mathematical Analysis and Applications 124 (1987), no. 1, 290-291.

[4] Elliptic equations and decomposition, Computers \& Mathematics with Applications 15 (1988), no. 1, 65-67.

[5] _ Nonlinear Stochastic Systems Theory and Applications to Physics, Mathematics and Its Applications, vol. 46, Kluwer Academic, Dordrecht, 1989.

[6] Solving Frontier Problems of Physics: The Decomposition Method, Fundamental Theories of Physics, vol. 60, Kluwer Academic, Dordrecht, 1994.

[7] _ The Nikolaevskiy model for nonlinear seismic waves, Mathematical and Computer Modelling 22 (1995), no. 3, 81-82.

[8] G. Adomian and R. Meyers, Nonlinear transport in moving fluids, Applied Mathematics Letters 6 (1993), no. 5, 35-38.

[9] G. Adomian and R. Rach, Equality of partial solutions in the decomposition method for linear or nonlinear partial differential equations, Computers \& Mathematics with Applications 19 (1990), no. $12,9-12$.

[10] Noise terms in decomposition solution series, Computers \& Mathematics with Applications 24 (1992), no. 11, 61-64.

[11] G. Adomian, R. Rach, and R. Meyers, An efficient methodology for the physical sciences, Kybernetes 20 (1991), no. 7, 24-34.

[12] K. Al-Khaled, Numerical solutions of the Laplace's equation, Applied Mathematics and Computation 170 (2005), no. 2, 1271-1283.

[13] J. Biazar and A. R. Amirtaimoori, An analytic approximation to the solution of heat equation by Adomian decomposition method and restrictions of the method, Applied Mathematics and Computation 171 (2005), no. 2, 738-745.

[14] J. Biazar and R. Islam, Solution of wave equation by Adomian decomposition method and the restrictions of the method, Applied Mathematics and Computation 149 (2004), no. 3, 807-814.

[15] O. R. Burggraf, An exact solution of the inverse problem in heat conduction theory and applications, ASME Journal of Heat Transfer 86C (1964), 373-382.

[16] P. J. Caudrey, I. C. Eilbeck, and J. D. Gibbon, The sine-Gordon equation as a model of classical field theory, Nuovo Cimento 25 (1975), 497-511.

[17] B. K. Datta, A new approach to the wave equation - an application of the decomposition method, Journal of Mathematical Analysis and Applications 142 (1989), no. 1, 6-12.

[18] _ A technique for approximate solutions to Schrödinger-like equations, Computers \& Mathematics with Applications 20 (1990), no. 1, 61-65.

[19] G. de Mey, S. de Smet, and M. Driscart, Forced convection cooling of flat electronic substrates, Boundary Element Technology VI (C. A. Brebbia, ed.), Computational Mechanics, Southampton, 1991, pp. 39-50.

[20] L. Debnath, Nonlinear Partial Differential Equations for Scientists and Engineers, 2nd ed., Birkhäuser, Massachusetts, 2005.

[21] E. Y. Deeba and S. A. Khuri, A decomposition method for solving the nonlinear Klein-Gordon equation, Journal of Computational Physics 124 (1996), no. 2, 442-448.

[22] S. M. El-Sayed, The decomposition method for studying the Klein-Gordon equation, Chaos, Solitons and Fractals 18 (2003), no. 5, 1025-1030. 
[23] S. M. El-Sayed and D. Kaya, Comparing numerical methods for Helmholtz equation model problem, Applied Mathematics and Computation 150 (2004), no. 3, 763-773.

[24] M. A. Golberg, A note on the decomposition method for operator equations, Applied Mathematics and Computation 106 (1999), no. 2-3, 215-220.

[25] M. Inc and Y. Cherruault, A new approach to travelling wave solution of a fourth-order semilinear diffusion equation, Kybernetes 32 (2003), no. 9-10, 1492-1503.

[26] _ A reliable approach to the Korteweg-de Vries equation: an application of the decomposition method, Kybernetes 34 (2005), no. 7-8, 951-959.

[27] M. Inc, Y. Cherruault, and K. Abbaoui, A computational approach to the wave equations: an application of the decomposition method, Kybernetes 33 (2004), no. 1, 80-97.

[28] D. Kaya, On the solution of a Korteweg-de Vries like equation by the decomposition method, International Journal of Computer Mathematics 72 (1999), no. 4, 531-539.

[29] _ An application of the decomposition method for second order wave equations, International Journal of Computer Mathematics 75 (2000), no. 2, 235-245.

[30] An explicit and numerical solutions of some fifth-order KdV equation by decomposition method, Applied Mathematics and Computation 144 (2003), no. 2-3, 353-363.

[31] D. Kaya and A. Yokus, A numerical comparison of partial solutions in the decomposition method for linear and nonlinear partial differential equations, Mathematics and Computers in Simulation 60 (2002), no. 6, 507-512.

[32] D. Lesnic, The decomposition method for Cauchy advection-diffusion problems, Computers \& Mathematics with Applications 49 (2005), no. 4, 525-537.

[33] D. Lesnic and L. Elliott, The decomposition approach to inverse heat conduction, Journal of Mathematical Analysis and Applications 232 (1999), no. 1, 82-98.

[34] J. D. Murray, Mathematical Biology, Biomathematics, vol. 19, Springer, Berlin, 1993.

[35] T. Myint-U and L. Debnath, Linear Partial Differential Equations for Scientists and Engineers, 4th ed., Birkhäuser, Massachusetts, 2006.

[36] S. Pamuk, An application for linear and nonlinear heat equations by Adomian's decomposition method, Applied Mathematics and Computation 163 (2005), no. 1, 89-96.

[37] V. Seng, K. Abbaoui, and Y. Cherruault, Adomian's polynomials for nonlinear operators, Mathematical and Computer Modelling 24 (1996), no. 1, 59-65.

[38] W. R. Smith, G. C. Wake, J. E. A. McIntosh, R. P. McIntosh, M. Pettigrew, and R. Kao, Mathematical analysis of perifusion data: models predicting elution concentration, American Journal of Physiology 261 (1991), R247-R256.

[39] A. Soufyane and M. Boulmalf, Solution of linear and nonlinear parabolic equations by the decomposition method, Applied Mathematics and Computation 162 (2005), no. 2, 687-693.

[40] A.-M. Wazwaz, Equality of partial solutions in the decomposition method for partial differential equations, International Journal of Computer Mathematics 65 (1997), no. 3-4, 293-308.

[41] Necessary conditions for the appearance of noise terms in decomposition solution series, Applied Mathematics and Computation 81 (1997), no. 2-3, 265-274.

[42] __ A reliable technique for solving the wave equation in an infinite one-dimensional medium, Applied Mathematics and Computation 92 (1998), no. 1, 1-7.

[43] - A reliable modification of Adomian decomposition method, Applied Mathematics and Computation 102 (1999), no. 1, 77-86.

[44] - An analytic study on the third-order dispersive partial differential equations, Applied Mathematics and Computation 142 (2003), no. 2-3, 511-520.

[45] A.-M. Wazwaz and A. Gorguis, Exact solutions for heat-like and wave-like equations with variable coefficients, Applied Mathematics and Computation 149 (2004), no. 1, 15-29.

[46] G. B. Whitham, Linear and Nonlinear Waves, John Wiley \& Sons, New York, 1974. 
[47] C. Zhao, Algorithm and mechanization to Cauchy problem of parabolic equation, Applied Mathematics and Computation 169 (2005), no. 2, 1038-1044.

[48] _ Algorithmization formula and mechanization to Cauchy problem of high dimensional wave equation, Applied Mathematics and Computation 167 (2005), no. 2, 957-963.

D. Lesnic: Department of Applied Mathematics, University of Leeds, Leeds LS2 9JT, UK

E-mail address: amt5ld@maths.leeds.ac.uk 


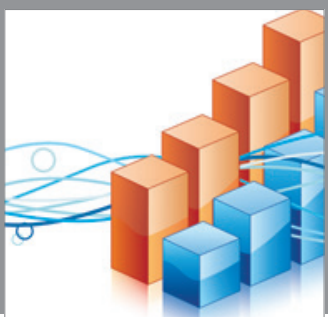

Advances in

Operations Research

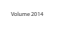

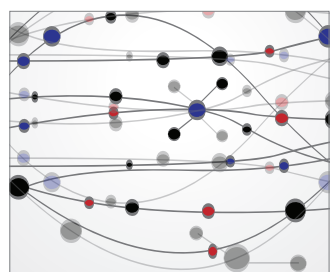

\section{The Scientific} World Journal
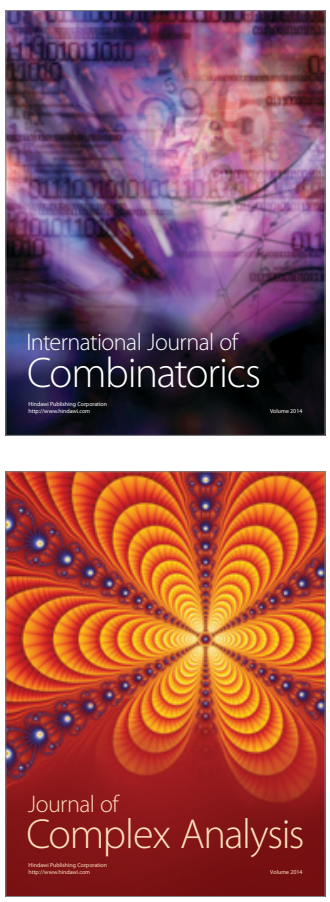

International Journal of

Mathematics and

Mathematical

Sciences
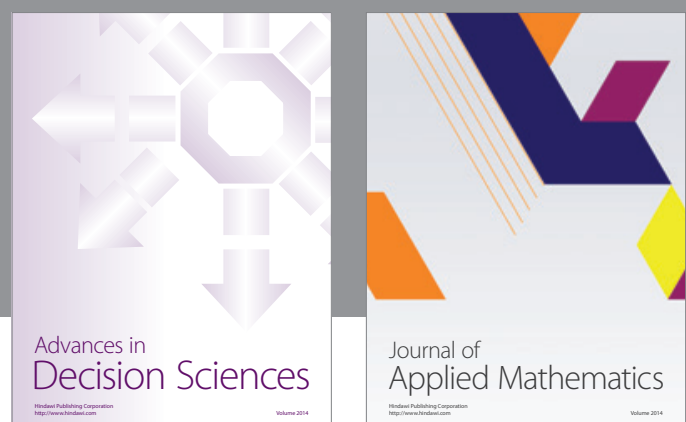

Journal of

Applied Mathematics
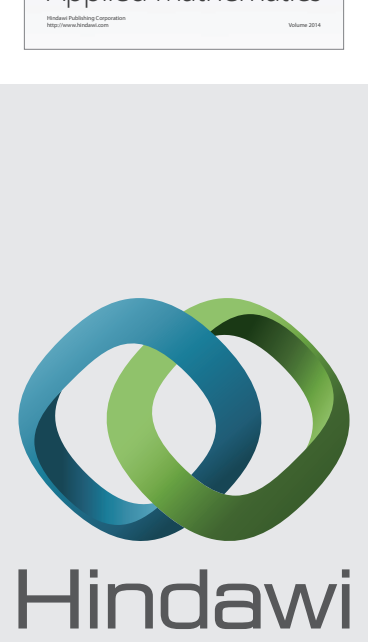

Submit your manuscripts at http://www.hindawi.com
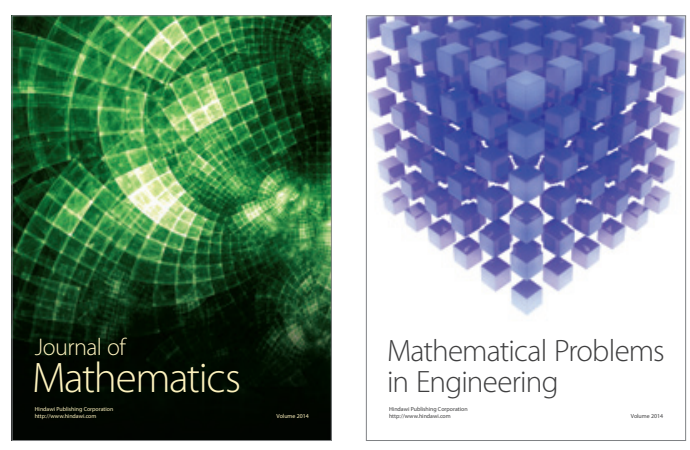

Mathematical Problems in Engineering
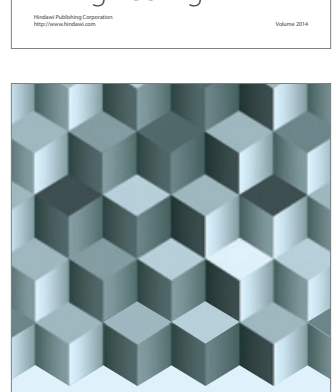

Journal of

Function Spaces
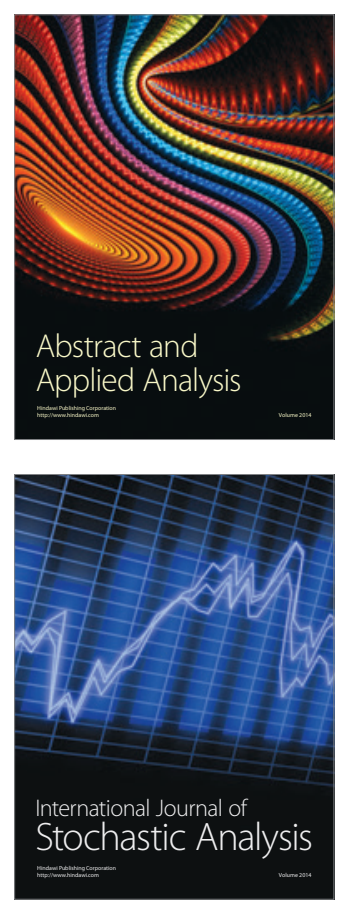

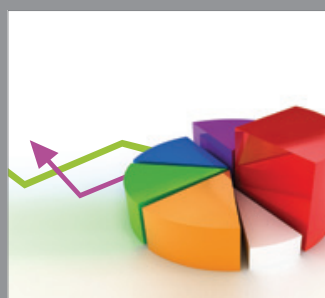

ournal of

Probability and Statistics

Promensencen
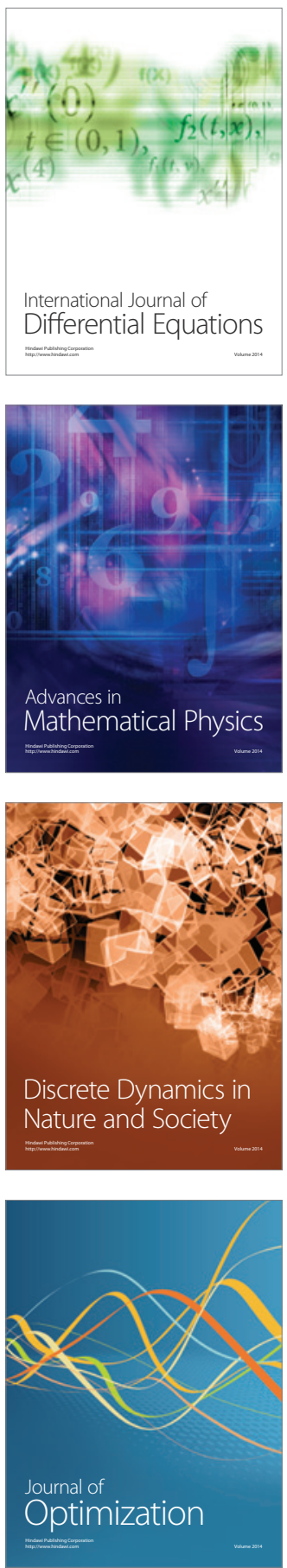